\title{
Representing preferences by Choquet integral: Guidelines to specify the capacity type
}

\author{
Leman Esra Dolgun ${ }^{a^{*}}$, Nimetullah Burnak ${ }^{\mathrm{b}}$ and Gulser Koksal ${ }^{\mathrm{c}}$
}

\begin{abstract}
${ }^{a}$ Department of Industrial Engineering, Eskișehir Technical University, 26555, Eskișehir, Turkey
${ }^{b}$ Department of Industrial Engineering, Eskişehir Osmangazi University, 26480, Eskişehir, Turkey

${ }^{c}$ Department of Industrial Engineering, Middle East Technical University, 06800, Ankara, Turkey

\begin{tabular}{l}
\hline C H R O N I C L E \\
\hline Article history: \\
Received March 23, 2020 \\
Received in revised format: \\
March 29, 2020 \\
Accepted April 222020 \\
Available online \\
April 22, 2020 \\
\hline Keywords: \\
Multiple criteria decision making \\
Choquet integral \\
Interaction \\
Unipolar capacity \\
K-ary capacity \\
Non-additive measures
\end{tabular}
A B S T R A C T

This study considers representing decision maker preferences by Choquet integral in existence of interactions among criteria. Parameters of the Choquet integral are capacities which assign weights not only to criteria but also to each subset of criteria. This property provides Choquet integral with the ability of modeling some types of interactions. Different capacity types with different degrees of complexity have been defined in the literature. After making a review on the dependence (interaction) and independence concepts used in the multiple criteria decision making literature, we study and represent structures of interactions that can be handled by different capacity types through intuitive graphical demonstrations. Afterwards, we provide guidelines for specifying the appropriate capacity type in practical applications. Such guidance has not been provided in the literature for the practitioners to the best of our knowledge.
\end{abstract}

Non-additive measures

(C) 2020 by the authors; licensee Growing Science, Canada.

\section{Introduction}

In this study, we consider the problem of constructing a value function that represents Decision Maker (DM) preferences from a set of decision examples on some reference alternatives in the existence of interactions between criteria. Throughout the paper we consider a finite set of alternatives $X$ evaluated on the basis of $n$ criteria $G=\left\{g_{1}, g_{2}, \ldots, g_{n}\right\}$ with the index set $N=\{1,2, \ldots, n\}$. Hence, the notation $\boldsymbol{x}=\left(x_{1}, x_{2}, \ldots, x_{n}\right)$ is used for any $\boldsymbol{x} \in X$. We use the index set to designate the criteria for convenience. $x_{i}^{j}$ denotes level $j$ (or $j$ th value) of criterion $i$. Existence of a common scale for criteria is assumed in the study. A value function, $v$, provides a numerical representation of preferences by aggregating all the criteria into a single performance measure defined such that:

$$
\begin{aligned}
& v(\boldsymbol{x})>v(\boldsymbol{y}) \Leftrightarrow \boldsymbol{x}>\boldsymbol{y} \\
& v(\boldsymbol{x})=v(\boldsymbol{y}) \Leftrightarrow \boldsymbol{x} \sim \boldsymbol{y}
\end{aligned}
$$

where $>$ and $\sim$ denote the preference and indifference relations, respectively (Doumpos \& Zopounidis, 2011). Such a value function defines an ordinal scale which means that there is no additional meaning of the values obtained by this function beyond the order that they imply (Dyer, 2005).

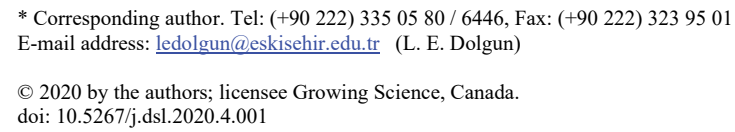


A value function which also involves comparison of preference differences between the alternatives, such that if $\boldsymbol{w}$ is preferred to $\boldsymbol{x}$ and $\boldsymbol{y}$ is preferred to $\boldsymbol{z}$, then the strength of preference for $\boldsymbol{w}$ over $\boldsymbol{x}$ is greater than or equal to the strength of preference for $\boldsymbol{y}$ over $\boldsymbol{z}$ if and only if the inequality given in Eq. (3) is satisfied, produces an interval scale (Dyer, 2005).

$v(\boldsymbol{w})-v(\boldsymbol{x}) \geq v(\boldsymbol{y})-v(\mathbf{z})$.

Once the value function is constructed, it can be used to rank, sort or classify the alternatives or to choose the best alternative. When constructing the value functions, most of the Multiple Criteria Decision Making (MCDM) methods assume independence of criteria and use weighted sum as the aggregation operator. Multiplicative value function of Multi-Attribute Value Theory (MAVT), Analytic Network Process (ANP) and Choquet integral are among the value function approaches that take into account interaction between criteria. Recently, there have been studies that combine ANP and Choquet integral (Ming-Lang et al., 2009; Gurbuz et al., 2012; Gurbuz \& Albayrak, 2014), combine TOPSIS with Choquet integral (Wang et al., 2019) and DEMATEL with Choquet integral (Abdullah et al., 2019). The literature also provides outranking approaches that consider interactions between criteria such as extensions of ELECTRE (Figueira et al., 2009) and PROMETHEE (Corrente et al., 2014) methods but still their capacities to handle all possible interactions are limited or not known to a full extent.

In this study, we focus on Choquet integral to represent DM preferences due to its ability to handle some interaction situations. The literature provides some examples to exhibit the weaknesses of the weighted sum and to demonstrate the ability of Choquet integral in modeling preferential interactions among criteria as detailed in Section 2. However, a scheme to clarify interaction situations that can be handled by different capacity types has not been provided. Therefore, in this study we aim to provide guidelines for the use of Choquet integral in possible cases of preferentially dependent criteria, i.e. for specifying the capacity type in practical applications. We utilize interaction graphs similar to the ones used in design of experiment studies to study and demonstrate interaction types that can be modeled by Choquet integral with respect to unipolar and k-ary capacities by concentrating on two way interactions. Such guidance has not been provided in the literature for the practitioners to the best of our knowledge. Moreover, applications of the k-ary capacity are represented which has only been studied in the literature theoretically to the best of our knowledge.

The rest of the paper is organized as follows. Dependence (interaction) and independence concepts used in the MCDM literature are reviewed in Section 2. Section 3 summarizes the basic concepts for Choquet integrals with respect to the unipolar and k-ary capacities. In Section 4, we investigate and present the interaction structures that can be modeled by these capacity types. Preference examples that violate the independence conditions of MAVT, and a discussion on modeling of these preference

examples by Choquet integral are also presented in Section 4. Section 5 describes a framework proposed for analyzing interactions in practice. A real life example is presented in Section 6. Finally, Section 7 concludes the paper.

\section{Dependence and independence concepts in the MCDM literature}

This section reviews dependence and independence concepts used in MCDM and explains the motivation of this study.

MAVT defines conditions as preference independence, difference independence and weak difference independence to decompose the value function into additive and multiplicative forms. One can see Dyer (2005) for a complete discussion on these three independence concepts and the corresponding forms for a value function. 
Definition 1. Criterion subset $I$ is preferentially independent of the complementary subset $\bar{I}$ if $\left(x_{I}^{1}, x_{\bar{I}}^{1}\right) \gtrsim\left(x_{I}^{2}, x_{\bar{I}}^{1}\right)$ for any two levels of $I\left(x_{I}^{1}\right.$ and $\left.x_{I}^{2}\right)$ and for any level of $\bar{I}\left(x_{\bar{I}}^{1}\right)$ implies $\left(x_{I}^{1}, x_{\bar{I}}^{k}\right) \gtrsim$ $\left(x_{I}^{2}, x_{\bar{I}}^{k}\right)$ for all $x_{\bar{I}}^{k}$.

The preference independence condition imposes that orientation of preference for two alternatives does not depend on the values of the criteria which are held fixed.

Definition 2. Criterion subset $I$ is difference independent of the complementary subset $\bar{I}$ if for all $x_{I}^{1}$, $x_{I}^{2}$ such that $\left(x_{I}^{1}, x_{\bar{I}}^{1}\right) \gtrsim\left(x_{I}^{2}, x_{\bar{I}}^{1}\right)$ for some $x_{\bar{I}}^{1}$, then $v\left(x_{I}^{1}, x_{\bar{I}}^{1}\right)-v\left(x_{I}^{2}, x_{\bar{I}}^{1}\right)=v\left(x_{I}^{1}, x_{\bar{I}}^{k}\right)-v\left(x_{I}^{2}, x_{\bar{I}}^{k}\right)$ for any $x_{\bar{I}}^{k}$.

The difference independence condition imposes that degree of preference difference between two alternatives does not depend on the values of the criteria which are held fixed.

Definition 3. Criterion subset $I$ is weak difference independent of the complementary subset $\bar{I}$ if for any $x_{I}^{1}, x_{I}^{2}, x_{I}^{3}, x_{I}^{4}$ such that $v\left(x_{I}^{1}, x_{\bar{I}}^{1}\right)-v\left(x_{I}^{2}, x_{\bar{I}}^{1}\right) \geq v\left(x_{I}^{3}, x_{\bar{I}}^{1}\right)-v\left(x_{I}^{4}, x_{\bar{I}}^{1}\right)$ for some $x_{\bar{I}}^{1}$, then $v\left(x_{I}^{1}, x_{\bar{I}}^{k}\right)-v\left(x_{I}^{2}, x_{\bar{I}}^{k}\right) \geq v\left(x_{I}^{3}, x_{\bar{I}}^{k}\right)-v\left(x_{I}^{4}, x_{\bar{I}}^{k}\right)$ for any $x_{\bar{I}}^{k}$.

The weak difference independence condition requires that ordering of preference differences does not depend on the levels held fixed.

Grabisch and Labreuche (2005a) provide a more general independence definition and define the independence situation as a situation where satisfaction of each criterion brings its own contribution to the overall satisfaction. However, a DM can state that for two decision criteria satisfaction of only one of them produces almost the same effect with satisfaction of both. In this case, there is substitutiveness between these two criteria. Alternatively, satisfaction of only one criterion can create a very small effect on the preference of the DM compared with the satisfaction of both. In this case, there is complementarity between these two criteria. Two similar notions are veto and favor. A criterion is said to have veto effect if nonsatisfaction of this criterion entails a low global score and have favor effect if satisfaction of it entails a high global score (Grabisch \& Labreuche, 2008a). In practical applications criteria are usually substitutive, complementary or have a veto or favor effect to some extent.

Statistical dependencies such as correlation may also occur between criteria, and correlation information may affect attitude of the DM. As Angilella et al. (2010) presents as an example, a car could be evaluated with respect to maximum speed, acceleration and price. Since a car with a high maximum speed has often also a high price, simultaneous satisfaction of both criteria is uncommon, and hence the DM favors a car with a high maximum speed and a moderate price. Consequently, comprehensive importance or simultaneous effect of these two criteria is greater than sum of their importance when considered separately. In this case, there is synergy between maximum speed and price. These two criteria complement each other to some extent. There is redundancy (or negative synergy) between maximum speed and acceleration, since a car with high maximum speed often has also a good acceleration. If these two criteria are considered separately, the global evaluation will be overestimated for cars with high maximum speed and good acceleration. Therefore, even if these two criteria can be very important for the DM, their comprehensive importance is smaller than sum of their importance when considered separately. These two criteria present some degree of substitutiveness.

The examples presented in the literature to exhibit the weaknesses of the weighted sum and to motivate the use of Choquet integral are usually based on conditional relative importance of criteria (Grabisch and Labreuche, 2008a). Notion of conditional relative importance describes decision strategies in which relative importance of one criterion compared to another depends on the value of a third criterion. A classical example is the assessment of students with respect to three criteria: mathematics (M), statistics (S) and language skills (L). The applicants are supposed to have strong scientific skills, therefore M and $\mathrm{S}$ have a big importance to the program director. However, he/she does not wish to favor too much 
students who have a scientific profile with some flaws in L. Moreover, usually, students good at M are also good at $\mathrm{S}$ which means that these two criteria are somewhat redundant. As a consequence, for students good at $\mathrm{M}$, the director prefers a student good at $\mathrm{L}$ to one good at $\mathrm{S}$. In this example, relative importance of $\mathrm{L}$ compared to $\mathrm{S}$ depends on the satisfaction level in $\mathrm{M}$. Choquet integral with respect to unipolar capacity is able to model rules of the following type (Grabisch \& Labreuche, 2005a):

(R1): If $M$ is the best satisfied criteria, $\mathrm{L}$ is more important than $\mathrm{S}$.

(R2): If $\mathrm{M}$ is the worst satisfied criteria, $\mathrm{S}$ is more important than $\mathrm{L}$.

Above rules specify the relative importance of criteria L and S only for the two extreme levels of performance (best and worst, respectively) and do not include intermediate values on criterion M. Rules $\left(\mathrm{R} 1^{\prime}\right)$ and (R2') specify the preferences between criteria $\mathrm{S}$ and $\mathrm{L}$ for all values of criterion $M$ and thus generalize (R1) and (R2):

$\left(\mathrm{R} 1^{\prime}\right)$ : For a student good at M, L is more important than S.

$\left(\mathrm{R} 2^{\prime}\right)$ : For a student bad at M, S is more important than $\mathrm{L}$.

$\left(\mathrm{R} 1^{\prime}\right)$ and (R2') make a reference to a neutral value (for example, 10) such that better values are considered as good whereas worse values are judged as bad by the DM. However, Choquet integral with respect to unipolar capacity does not allow modeling rules that make a reference to absolute values. On the other hand, Labreuche and Grabisch (2007) show that Choquet integral with respect to bicapacity enables modeling rules of the following type:

$\left(\mathrm{R} 1{ }^{\prime \prime}\right)$ : If the score with respect to $\mathrm{M}$ is good and it is not the closest one to the neutral level among the three criteria, then criterion $\mathrm{L}$ is more important than criterion $\mathrm{S}$.

(R2"): If the score with respect to $\mathrm{M}$ is bad and it is not the closest one to the neutral level among the three criteria, then criterion $\mathrm{S}$ is more important than criterion $\mathrm{L}$.

Although there are three reference levels in the student assessment example (as 0, 10, and 20) there can be other decision situations modeling of which require specification of more reference levels since decision strategy of the DM is different at different regions defined by these reference levels. For example, in manufacturing domain, value of a product's quality characteristic is usually classified as scrap, rework and acceptable and decision strategy of the quality control expert can be different at these regions such as the following: if value of one of the quality characteristics is at the scrap region, then this quality characteristic has a veto effect whereas if all of the quality characteristics are at the acceptable region, then each of them brings its own contribution to the overall quality value of the item under consideration. In health care, as another example, certain values of a patient deemed as critical may necessitate narrowing intervals for acceptable values for other indicators. Choquet integral with respect to k-ary capacity can be useful in modeling such decision strategies as illustrated in this current study.

Choquet integral literature provides some examples to demonstrate the ability of Choquet integral to model preferential interactions as explained in the previous paragraphs. However, a scheme to clarify interaction situations that can be handled by different capacity types has not been provided. Therefore, in this study we utilize intuitive interaction graphs similar to the ones used in design of experiment studies to study and demonstrate interaction types that can be modeled by Choquet integral with respect to unipolar and k-ary capacities by concentrating on two way interactions.

\section{Choquet Integral with respect to unipolar and k-ary capacities}

Capacity (Choquet, 1953) or fuzzy measure (Sugeno, 1974) is a set function $\mu: 2^{N} \rightarrow[0,1]$ satisfying the following conditions:

- $A \subseteq B \subseteq N \Rightarrow \mu(A) \leq \mu(B)$ (monotonicity conditions), 
- $\mu(\varnothing)=0, \mu(N)=1$ (boundary conditions).

Choquet integral for $\boldsymbol{x}=\left(x_{1}, x_{2}, \ldots, x_{n}\right) \in R_{+}^{n}$ with respect to a unipolar capacity $\mu$ is given by

$C_{\mu}(\boldsymbol{x})=\sum_{i=1}^{n}\left[x_{\sigma(i)}-x_{\sigma(i-1)}\right] \mu(\{\sigma(i), \ldots, \sigma(n)\})$

where $\sigma$ is a permutation on $N$ such that $x_{\sigma(1)} \leq x_{\sigma(2)} \leq \cdots \leq x_{\sigma(n)}$ and $x_{\sigma(0)}=0$. An equivalent representation is

$C_{\mu}(\boldsymbol{x})=\sum_{i=1}^{n} x_{\sigma(i)}[\mu(\{\sigma(i), \ldots, \sigma(n)\})-\mu(\{\sigma(i+1), \ldots, \sigma(n)\})]$

with the convention $\{\sigma(n+1)\}=\emptyset$.

It can be noted that $C_{\mu}\left(1_{A}, 0_{-A}\right)=\mu(A), \forall A \in N$ where the notation $\boldsymbol{x}=\left(1_{A}, 0_{-A}\right)$ stands for an alternative defined by $x_{i}=1$ if $i \in A$ and $x_{i}=0$, otherwise. Therefore, $\mu(A)$ is interpreted as the overall assessment of the binary alternative $\left(1_{A}, 0_{-A}\right)$ (Grabisch and Labreuche, 2005a). Hence the binary vector $\left(1_{A}, 0_{-A}\right)$ is used as the prototype alternative (vector) for the Choquet integral with respect to unipolar capacity and the whole model is constructed using these prototype alternatives. Elicitation of unipolar capacity values (i.e. elicitation of preference scores for the binary alternatives $\left(1_{A}, 0_{-A}\right), \forall A \in N$ ) has been taken into account in several studies in the literature (Marichal and Roubens, 2000; Sicilia et al., 2003; Grabisch et al., 2008; Angilella et al., 2010, 2013; Timonin, 2013; Bottero et al., 2018). Angilella et al. (2004) and Angilella et al. (2015) consider specification of the common scale together with the capacity values compatible with the preference information provided by the DM.

A capacity is additive if for any $A, B \subseteq N$ such that $A \cap B=\emptyset$, we have $\mu(A \cup B)=\mu(A)+\mu(B)$. If $\mu$ is additive, Choquet integral reduces to the weighted sum.

Interaction index for criteria subset $A$ is defined as given by Equation (6) (Grabisch and Labreuche, 2005a).

$I(A)=\sum_{K \subseteq N \backslash A} \frac{(n-|K|-|A|) !|K| !}{(n-|A|+1) !} \sum_{L \subseteq A}(-1)^{|A|-|L|} \mu(K \cup L), \quad \forall A \subseteq N, A \neq \emptyset$,

$I(A)>0$ (resp. $<0,=0$ ) indicates complementarity (resp. substitutiveness, non-interaction) among criteria. A capacity is k-additive if $I(A)=0$ whenever $|A|>k$, and there exists some $A \subseteq N$ with $|A|=k$ such that $I(A) \neq 0$. A 2 -additive capacity allows modeling interaction between two criteria (Grabisch \& Labreuche, 2005a).

The capacity explained so far is called unipolar capacity since it considers only two reference levels as 0 and 1. Choquet integral is an aggregation operator that considers ordering of criteria values. Choquet integral with respect to unipolar capacity behaves like a weighted sum for alternatives having the same criteria rankings. This property is called comonotonic additivity (Grabisch and Labreuche, 2005a). Consequently, Choquet integral with respect to the unipolar capacity is not able to model rules that make a reference to absolute values. The literature presents more advanced capacity types as bi-capacity and k-ary capacity.

Bi-capacity allows considering ternary alternatives $\left(1_{A},-1_{B}, 0_{-(A \cup B)}\right)$, for all disjoint subsets $A, B$ of $N$ where 0 denotes the neutral level and -1 and 1 denote the least and greatest levels, respectively. Let 
$Q(N)=\left\{(A, B) \in 2^{N} \times 2^{N} \mid A \cap B=\emptyset\right\}$, a bi-capacity is a function $v: Q(N) \rightarrow R$ satisfying the following conditions (Grabisch and Labreuche, 2005b; Labreuche and Grabisch, 2007):

$$
\begin{array}{ll}
\text { - } & v(\varnothing, \emptyset)=0, v(N, \emptyset)=1 \text { and } v(\varnothing, N)=-1, \\
\text { - } & A \subseteq A^{\prime} \text { implies } v(A, B) \leq v\left(A^{\prime}, B\right), \\
\text { - } & B \subseteq B^{\prime} \text { implies } v(A, B) \geq v\left(A, B^{\prime}\right) .
\end{array}
$$

Choquet integral for $\boldsymbol{x}=\left(x_{1}, x_{2}, \ldots, x_{n}\right) \in R^{n}$ with respect to bi-capacity $v$ is defined as follows (Grabisch and Labreuche, 2005c):

$B C_{v}(\boldsymbol{x})=C_{\mu_{N^{+}, N^{-}}}(|\boldsymbol{x}|)$

where $\mu_{N^{+}, N^{-}}(A)=v\left(A \cap N^{+}, A \cap N^{-}\right), N^{+}=\left\{i \in N \mid x_{i} \geq 0\right\}$ and $N^{-}=N \backslash N^{+}$.

An example illustrating the application of Eq. (7) is given in Appendix A.

Readers are referred to Grabisch and Labreuche (2005a) and Grabisch and Labreuche (2008a) for comprehensive reviews of Choquet integral with respect to unipolar capacity and bi-capacity in MCDM.

The Choquet integral with respect to a capacity can be obtained as a unique parsimonious (i.e., using the least number of vertices, $n+1$ ) linear interpolator between vertices of the hypercube $[0,1]^{n}$ (Grabisch, 2004). The vertices used in the interpolation formula are obtained by partitioning (or, triangulation of) the hypercube into $q=n$ ! polyhedral defined by their set of vertices $V_{1}, V_{2}, \ldots, V_{q}$, all vertices being vertices of $[0,1]^{n}$. The convex hull of the vertices used for the interpolation $\operatorname{conv}(V(x))$ for given $\boldsymbol{x} \in[0,1]^{n}$ contains $\boldsymbol{x}$, and any $\boldsymbol{x} \in[0,1]^{n}$ belongs to a unique polyhedron (except for common frontiers). Continuity should also be ensured. Each polyhedron is defined by a permutation $\sigma$ on $N: \operatorname{conv}\left(V_{\sigma}\right)=\left\{\boldsymbol{x} \in[0,1]^{n} \mid x_{\sigma(1)} \leq \cdots \leq x_{\sigma(n)}\right\}$.

The interpolation formula was discovered by Lovász (1983) by considering the problem of extending the domain of pseudo-Boolean function from $\{0,1\}^{n}$ to $[0,1]^{n}$. Marichal $(2002)$ observed that the interpolation formula was precisely the Choquet integral (Grabisch and Labreuche, 2008b). Grabisch and Labreuche (2008b) used the idea of considering Choquet integral as a parsimonious linear interpolator as a basic principle for extending the notion of Choquet integral to more general frameworks. An example of this is considering multiple reference levels in the context of multicriteria aggregation (such as using k-ary capacities).

For criterion $i$, let us denote by $L_{i}$ the set of reference levels, assuming they are partially ordered by some order relation $\leq_{i}$ such that $\left(L_{i}, \leq_{i}\right)$ is a distributive lattice. The product lattice $L=L_{1} \times \ldots \times L_{n}$ represents the possible combinations of all reference levels for all criteria. The geometric realization of $L$ gives all possible values the criteria can receive. The idea is that the output of aggregation is known for each element of $L$, and the output for any point in the geometric realization of $L$ is obtained as a linear interpolation between vertices defined by the natural triangulation.

Readers are referred to Grabisch and Labreuche (2003) and Grabisch and Labreuche (2008b) for detailed explanations on the concepts of geometric realization of a distributive lattice and its natural triangulation and extension of these concepts to the bipolar structure. Here we only give definition of the k-ary capacity and Choquet integral formulation with respect to the k-ary capacity based on Grabisch and Labreuche (2003).

A k-ary capacity is a set function defined on the lattice $L=k^{n}$ representing overall score of k-ary alternative $\left(\alpha_{A_{q}^{-}}^{-q}, \alpha_{A_{q-1}^{-}}^{-(q-1)}, \ldots, \alpha_{A_{1}^{-}}^{-1}, \alpha_{A_{1}^{+}}^{1}, \ldots, \alpha_{A_{p-1}^{+}}^{p-1}, \alpha_{A_{p}^{+}}^{p}\right)$ for all disjoint subsets $A_{q}^{-}, A_{q-1}^{-}, \ldots, A_{1}^{-}, A_{1}^{+}$, 
$\ldots, A_{p-1}^{+}, A_{p}^{+}$of $N$ where $\alpha=\left\{\alpha^{-q}, \alpha^{-(q-1)}, \ldots, \alpha^{-1}, \alpha^{0}, \alpha^{1}, \ldots, \alpha^{p-1}, \alpha^{p}\right\}$ represents the set of reference levels with $\alpha^{0}=0, \alpha^{-j}<0, \forall j \in\{1,2, \ldots, q\}, \alpha^{j}>0, \forall j \in\{1,2, \ldots, p\}$ and $q+p+1=k$.

Unipolar capacity is 2-ary capacity based on reference levels 0 and 1 while bi-capacity is 3-ary capacity based on reference levels $-1,0$ and 1 .

Choquet integral for $\boldsymbol{x}=\left(x_{1}, x_{2}, \ldots, x_{n}\right) \in R^{n}$ with respect to a k-ary capacity $\mu$ is defined as follows:

$K C_{\mu}(\boldsymbol{x})=C_{\mu_{\mathcal{A}}}(\Phi(\boldsymbol{x}))$

where

$\mathcal{A}=\left(A_{q}^{-}, A_{q-1}^{-}, \ldots, A_{1}^{-}, A_{1}^{+}, \ldots, A_{p-1}^{+}, A_{p}^{+}\right)$,

$\mu_{\mathcal{A}}(B)=\mu\left(B_{q}^{-}, \ldots, B_{p}^{+}\right)$,

$B_{j}^{+}=\left(A_{j}^{+} \cap B\right) \cup\left(A_{j+1}^{+} \backslash B\right), j=1, \ldots, p, A_{p+1}^{+}=\emptyset$ and $B_{j}^{-}=\left(A_{j}^{-} \cap B\right) \cup\left(A_{j+1}^{-} \backslash B\right), j=1, \ldots, q$, $A_{q+1}^{-}=\varnothing$,

$i \in A_{j}^{+}$if $x_{i} \in\left(\alpha^{j-1}, \alpha^{j}\right]$ and $i \in A_{j}^{-}$if $x_{i} \in\left[\alpha^{-j}, \alpha^{-j+1}\right)$.

$C_{\mu_{\mathcal{A}}}(\mathbf{y})=\mu_{\mathcal{A}}(\emptyset)+\sum_{i=1}^{n} y_{\sigma(i)}\left[\mu_{\mathcal{A}}(\{\sigma(i), \ldots, \sigma(n)\})-\mu_{\mathcal{A}}(\{\sigma(i+1), \ldots, \sigma(n)\})\right]$

where $\mathbf{y}=\Phi(\boldsymbol{x})$.

The function $\Phi:\left[\alpha^{-q}, \alpha^{p}\right]^{n} \rightarrow[0,1]^{n}$ is defined as follows:

$\Phi_{i}(\boldsymbol{x})=\left\{\begin{array}{c}\frac{x_{i}-\alpha^{j-1}}{\alpha^{j}-\alpha^{j-1}} \text { if } i \in A_{j}^{+} \\ \frac{\left|x_{i}-\alpha^{-(j-1)}\right|}{\alpha^{-(j-1)}-\alpha^{-j}} \text { if } i \in A_{j}^{-}\end{array}\right.$

The Choquet integral formulation with respect to 5 -ary capacity with reference values $-1,-0.5,0$, 0.5 , and 1 for an alternative $\boldsymbol{x}=(-0.85,0.65,0.4)$ is given in Appendix B.

Notations used in this study for reference levels and the resulting regions are shown in Fig. 1. The notations shown in Fig. 1 are used interchangeably in the rest of the paper.

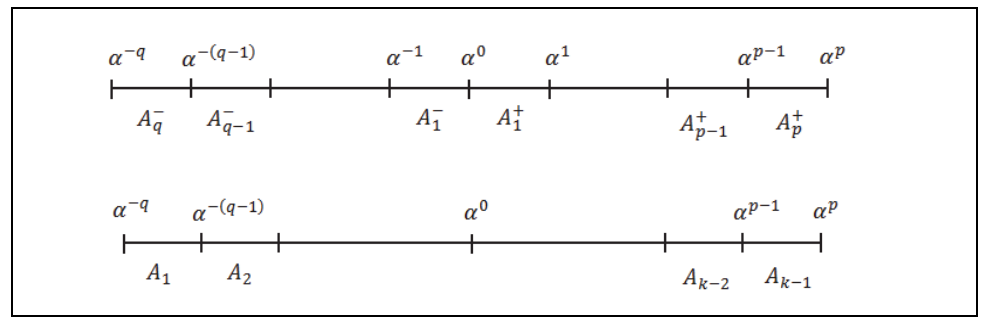

Fig. 1. Notations used for reference levels and the resulting regions

\section{Investigation of interaction types that can be modeled by Choquet Integral}

In this study, we concentrate on two-way interactions. We utilize interaction graphs that are used in design of statistical experiment studies to demonstrate the interaction types that can be modeled by Choquet integral. 
Definition 4. Two criteria 1 and 2 are said to interact if the change in preference for a 1-unit change in criterion 1, when criterion 2 is held fixed, depends on the value of criterion 2 (adapted from Montgomery (2005)).

In Fig. 2(a), effect of criterion 1 on preference is the same regardless of the level of criterion 2 and the effect of criterion 2 is the same for all levels of criterion 1 . If this situation is valid for all possible levels, these two criteria can be deemed as independent.

Fig. 2(b) and Fig. (c) illustrate interaction cases. In Figure 2(b), effect of criterion 1 is larger when criterion 2 is at its second level. Therefore, preference difference between the levels of criterion 1 changes at different levels of criterion 2 (difference independence condition of MAVT is not satisfied). This situation is called as "change in preference difference" in this study. In Figure 2(c), although changing criterion 1 from its first level to the second level creates a positive effect on the preference when criterion 2 is at its second level, criterion 1 has a negative effect when criterion 2 is at its first level (preferential independence condition of MAVT is not satisfied). This case, where preference direction changes, is referred to as "change in preference direction" in this study.

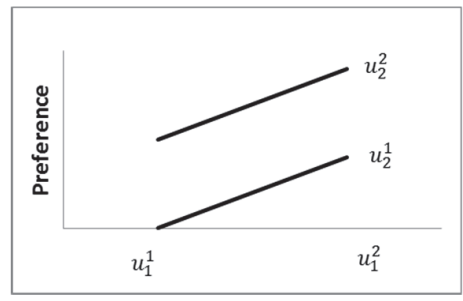

(a)

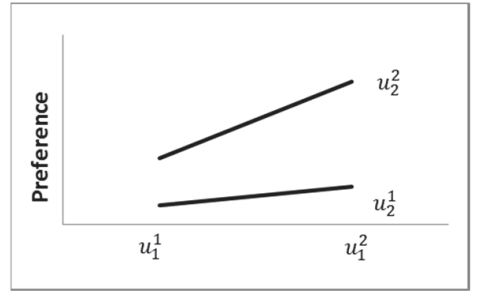

(b)

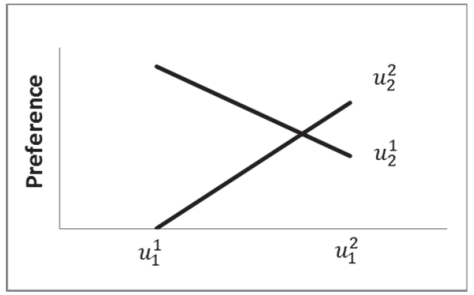

(c)

Fig. 2. Interaction plots. (a) No interaction. (b) Change in preference difference. (c) Change in preference direction. $u_{i}$ is used to denote the value of criterion $i$ transformed to interval $[0,1]$.

In Sections 4.1 and 4.2, Choquet integrals with respect unipolar capacity and k-ary capacity are investigated, respectively, in terms of their ability to model different interaction situations. Conditions that the Choquet integral model requires to represent the preferences are revealed by concentrating on change in preference difference. Discussions on representation of preferences that violate difference independence and weak difference independence conditions of MAVT are provided.

In the rest of the paper, $u_{i}$ is used to denote the value of criterion $i$ transformed to interval $[0,1]$. For the unipolar capacity case, $u_{i}=x_{i}$, and $u_{i}=\left|x_{i}\right|$ for the bi-capacity case. The transformation is performed via Equation (10) for the k-ary capacity case.

\subsection{Interaction structures that can be modeled by unipolar capacity}

With the purpose of specifying the conditions that can be modeled by unipolar capacity, preference difference created by changing criterion 1 from one of its levels to another, i.e. $d$ in Fig. 3, is investigated for changing levels of criterion 2 based on the fact that Choquet integral is an aggregation operator that considers ordering of criteria values. If there are more than two criteria, they are assumed to be fixed at some appropriate levels.

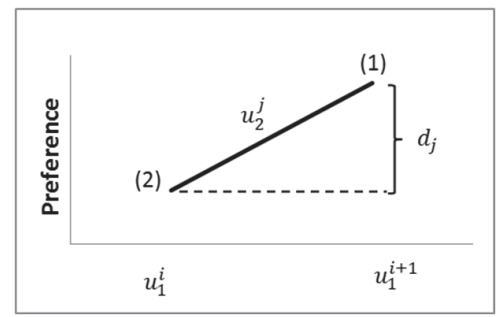

Fig. 3. Preference difference created by changing criterion 1 from one of its levels to another 
In Fig. $3, u_{1}^{i}$ and $u_{1}^{i+1}$ denote any two values of criterion 1 such that $u_{1}^{i}<u_{1}^{i+1}$ and $u_{2}^{j}$ is any value of criterion 2. For the sake of simplicity it is assumed that the other criteria of the problem are kept at their worst levels and hence take the value of zero. Then, the preference difference, $d_{j}$, of the two alternatives $\left(u_{1}^{i+1}, u_{2}^{j}, 0_{N \backslash\{1,2\}}\right)$ and $\left(u_{1}^{i}, u_{2}^{j}, 0_{N \backslash\{1,2\}}\right)$, denoted as (1) - (2) in Fig. 3, is given by Eq. (11) ( $i$ is taken as 1$)$ :

$$
d_{j}=\left\{\begin{array}{l}
\left(u_{1}^{2}-u_{1}^{1}\right)[\mu(1)-\mu(\varnothing)], \text { if } u_{2}^{j} \leq u_{1}^{1}<u_{1}^{2} \\
\left(u_{2}^{j}-u_{1}^{1}\right)[\mu(1,2)-\mu(2)]+\left(u_{1}^{2}-u_{2}^{j}\right)[\mu(1)-\mu(\varnothing)], \text { if } u_{1}^{1}<u_{2}^{j}<u_{1}^{2} \\
\left(u_{1}^{2}-u_{1}^{1}\right)[\mu(1,2)-\mu(2)], \text { if } u_{1}^{1}<u_{1}^{2} \leq u_{2}^{j}
\end{array}\right.
$$

Proof of Equation (11) is given in Appendix C. Now we investigate $d_{j}$ for changing levels of criterion 2 such that $u_{2}^{1}<u_{2}^{2}<\cdots<u_{2}^{j}<\cdots<u_{2}^{l_{2}}$.

First, consider that $u_{2}^{1}<u_{1}^{1}<u_{1}^{2}$. Then, $d_{1}$, the preference difference between the two alternatives $\left(u_{1}^{2}, u_{2}^{1}, 0_{N \backslash\{1,2\}}\right)$ and $\left(u_{1}^{1}, u_{2}^{1}, 0_{N \backslash\{1,2\}}\right)$, equals to $\left(u_{1}^{2}-u_{1}^{1}\right)[\mu(1)-\mu(\varnothing)]$ from Eq. (11). The preference difference will be equal to $d_{1}$ for all alternative pairs for which the ranking $u_{2}^{j}<u_{1}^{1}<u_{1}^{2}$ is satisfied. Slope of the line connecting preference values of the alternatives $\left(u_{1}^{2}, u_{2}^{1}, 0_{N \backslash\{1,2\}}\right)$ and $\left(u_{1}^{1}, u_{2}^{1}, 0_{N \backslash\{1,2\}}\right)$ will be equal to $[\mu(1)-\mu(\varnothing)]$.

Let us assume that $d_{2}$ is the preference difference for the case $u_{1}^{1}<u_{2}^{2}<u_{1}^{2}$. Then, $d_{2}=$ $\left(u_{2}^{2}-u_{1}^{1}\right)[\mu(1,2)-\mu(2)]+\left(u_{1}^{2}-u_{2}^{2}\right)[\mu(1)-\mu(\varnothing)]$ from Eq. (11). Having $d_{1}>d_{2}$ implies that $[\mu(1)-\mu(\emptyset)]>[\mu(1,2)-\mu(2)]$.

Proceeding in a similar manner, suppose that $d_{3}$ is the preference difference between two alternatives $\left(u_{1}^{2}, u_{2}^{3}, 0_{N \backslash\{1,2\}}\right)$ and $\left(u_{1}^{1}, u_{2}^{3}, 0_{N \backslash\{1,2\}}\right)$, where ranking of criteria values is $u_{1}^{1}<u_{2}^{3}<u_{1}^{2}$. It can be seen from Equation (11) that $\left.d_{3}=\left(u_{2}^{3}-u_{1}^{1}\right)[\mu(1,2)-\mu(2)]+\left(u_{1}^{2}-u_{2}^{3}\right)[\mu(1)-\mu(\varnothing)]\right)$. Since $d_{2}-$ $d_{3}=\left(u_{2}^{3}-u_{2}^{2}\right)\{[\mu(1)-\mu(\varnothing)]-[\mu(1,2)-\mu(2)]\}$, previous condition of $d_{1}>d_{2}$ imposes that this difference is positive, i.e., $d_{3}$ is smaller than $d_{2}$.

Similarly, $d_{4}$, preference difference between the alternatives $\left(u_{1}^{2}, u_{2}^{4}, 0_{N \backslash\{1,2\}}\right)$ and $\left(u_{1}^{1}, u_{2}^{4}, 0_{N \backslash\{1,2\}}\right)$ where $u_{1}^{1}<u_{1}^{2}<u_{2}^{4}$, equals to $\left(u_{1}^{2}-u_{1}^{1}\right)[\mu(1,2)-\mu(2)]$. The slope of the line connecting preference values of these two alternatives is $\mu(1,2)-\mu(2)$. Since $d_{3}-d_{4}=\left(u_{1}^{2}-u_{2}^{3}\right)\{[\mu(1)-\mu(\varnothing)]-$ $[\mu(1,2)-\mu(2)]\}, d_{3}$ is greater than $d_{4}$. As a result, if $d_{1}>d_{2}$, then $d_{1}>d_{2}>d_{3}>d_{4}$. Note that if these two criteria are independent, then $\mu(1)-\mu(\varnothing)=\mu(1,2)-\mu(2)$ and $d$ values will be equal to each other, i.e. the corresponding lines will be parallel.

Equation (11) indicates that in the case of interaction, Choquet integral with respect to unipolar capacity produces a piecewise linear function for criterion 1 for each level of criterion 2 (other criteria are held fixed). Level of criterion 2 determines the break point of the function. Let us reconsider the $u_{1}^{1}<u_{2}^{2}<$ $u_{1}^{2}$ case. We will examine the interval $\left[u_{1}^{1}, u_{1}^{2}\right]$ in two parts as $\left[u_{1}^{1}, u_{2}^{2}\right]$ and $\left[u_{2}^{2}, u_{1}^{2}\right]$. The preference difference between the alternatives $\left(u_{1}^{1}, u_{2}^{2}, 0_{N \backslash\{1,2\}}\right)$ and $\left(u_{2}^{2}, u_{2}^{2}, 0_{N \backslash\{1,2\}}\right)$ (created by considering end points of the first interval: the end points constitute the values for criterion 1 while the value of criterion 2 is $\left.u_{2}^{2}\right)$ is $\left(u_{2}^{2}-u_{1}^{1}\right)[\mu(1,2)-\mu(2)]$ and the slope of the line connecting preference values of these two alternatives is $s_{1}=\mu(1,2)-\mu(2)$. The preference difference between the alternatives $\left(u_{2}^{2}, u_{2}^{2}, 0_{N \backslash\{1,2\}}\right)$ and $\left(u_{1}^{2}, u_{2}^{2}, 0_{N \backslash\{1,2\}}\right)$ is $\left(u_{1}^{2}-u_{2}^{2}\right)[\mu(1)-\mu(\varnothing)]$ and the slope of the line connecting preference values of the alternatives is $s_{2}=\mu(1)-\mu(\emptyset)$. 
The situation for $u_{1}^{1}<u_{2}^{3}<u_{1}^{2}$ is the same. If we investigate the $\left[u_{1}^{1}, u_{1}^{2}\right]$ interval in two parts as $\left[u_{1}^{1}, u_{2}^{3}\right]$ and $\left[u_{2}^{3}, u_{1}^{2}\right]$, the slope corresponding to the first part is $s_{1}=\mu(1,2)-\mu(2)$. And the slope for the second part is $s_{2}=\mu(1)-\mu(\emptyset)$.

Consequently, although the break points change, slopes of the first segments $\left(s_{1}\right)$ will be equal to each other for changing values of criterion 2 . The same is also true for the second segments $\left(s_{2}\right)$. Fig. 4 shows the preference lines for the cases discussed so far.

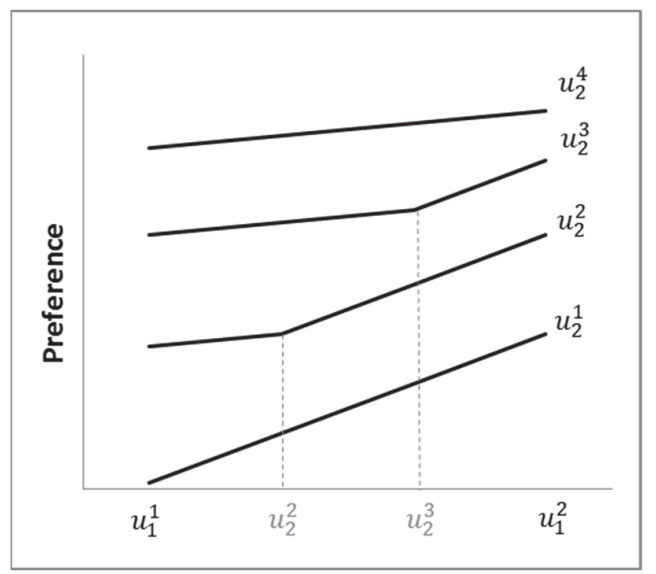

Fig. 4. Preference lines for the cases discussed in Section 4.1

The results are summarized in Table 1. Results 1 and 2 presented in Table 1 describe situations in which interaction cannot be modeled. These two results are in agreement with the comonotonic additivity property of the unipolar capacity. On the other hand, Results 3 and 4 show the structure of the interactions that can be modeled by Choquet integral with respect to unipolar capacity. Therefore, the aim of Table 1 is to guide practitioners in understanding whether their decision structures can be handled by the unipolar capacity and such guidance has not been provided clearly in the literature to the best of our knowledge.

In order to study interaction structures, we broadly divide interaction types into two as "change in preference difference" and "change in preference direction" in this study. Change in preference direction is related to violation of preferential independence whereas change in preference difference is related to violation of weak difference independence and/or difference independence of MAVT (Change in preference difference situation is also related to violation of preferential independence if it includes change in indifference situation).

As noted previously, the difference independence condition imposes that degree of preference difference between two alternatives does not depend on the values of the criteria which are held fixed, whereas the weak difference independence condition requires ordering of preference differences not to depend on the levels held fixed. Consequently, the difference independence condition of MAVT coincides with no interaction case. Let us consider a preference example given by Eq. (12) and Eq. (13) violating the difference independence condition.

$$
\begin{aligned}
& v(0.5,0.1)-v(0.2,0.1) \neq v(0.5,0.6)-v(0.2,0.6) \\
& v(0.7,0.1)-v(0.5,0.1) \neq v(0.7,0.6)-v(0.5,0.6)
\end{aligned}
$$

From Result 3, we know that these preference statements can be modeled by Choquet integral with unipolar capacity (since the positions of criterion 2 in the ranking with respect to criterion 1 are different for the alternatives in two sides of the inequalities). 
Table 1

Conditions required to model change in preference difference by unipolar capacity

Plot of preference differences

\section{Conditions required to model change in preference difference}

Result 1. Preference differences are equal to each other, i.e. $d_{j}=d_{j+1}=\cdots=d_{t}$, for all $u_{2}$ values satisfying the following ranking:

$$
u_{2}^{j}<u_{2}^{j+1}<\cdots<u_{2}^{t}<u_{1}^{i}<u_{1}^{i+1}
$$

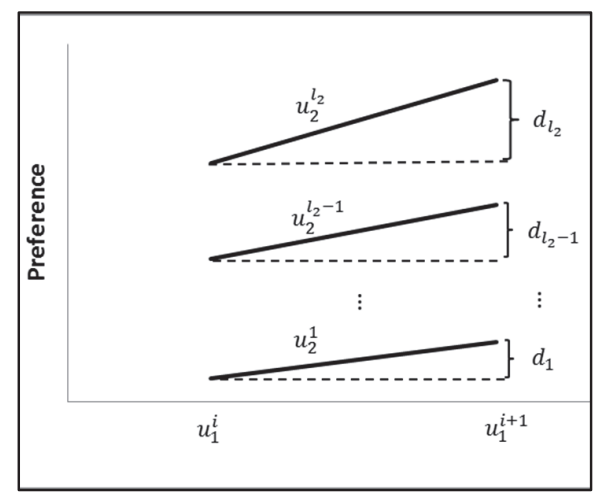

Result 2. Preference differences are equal to each other, i.e. $d_{j}=d_{j+1}=\cdots=d_{t}$, for all $u_{2}$ values satisfying the following ranking:

$$
u_{2}^{t}>\cdots>u_{2}^{j+1}>u_{2}^{j}>u_{1}^{i+1}>u_{1}^{i}
$$

Result 3. Preference differences increase or decrease monotonically or they are equal to each other. More precisely, one of the following three rankings is valid for all $u_{2}$ values which are not smaller than $u_{1}^{i}$ or larger than $u_{1}^{i+1}$ simultaneously (for example, $u_{2}^{1}<u_{1}^{i}<u_{2}^{2}<u_{2}^{3}<u_{1}^{i+1}<$ $\left.u_{2}^{4}\right)$

$$
\begin{aligned}
& -d_{l_{2}}>d_{l_{2}-1}>\cdots>d_{1} \\
& -d_{l_{2}}<d_{l_{2}-1}<\cdots<d_{1} \\
& -d_{l_{2}}=d_{l_{2}-1}=\cdots=d_{1}
\end{aligned}
$$

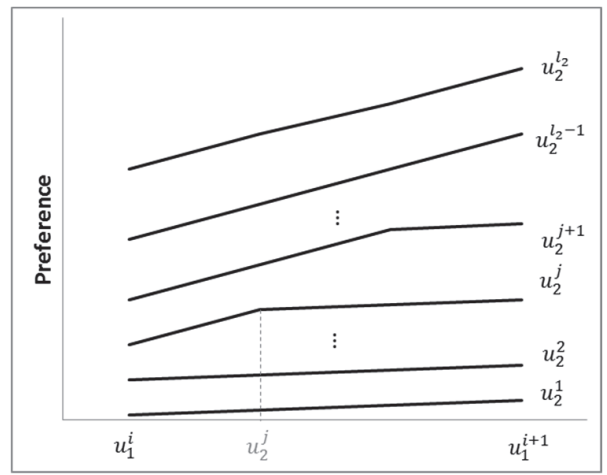

Result 4. In the case of interaction, Choquet integral with respect to unipolar capacity produces a piecewise linear function for criterion 1 for each level of criterion 2. The break point occurs at the level of criterion 2 . The adjacent figure illustrates this result for the ranking given below.

$$
u_{2}^{1}<u_{2}^{2}<u_{1}^{i}<u_{2}^{j}<u_{2}^{j+1}<u_{1}^{i+1}<u_{2}^{l_{2}-1}<u_{2}^{l_{2}}
$$

Suppose that for these eight alternatives the following preference structure is provided by the DM which violates weak difference independence condition.

$v(0.5,0.1)-v(0.2,0.1)>v(0.7,0.1)-v(0.5,0.1)$

$v(0.5,0.6)-v(0.2,0.6)<v(0.7,0.6)-v(0.5,0.6)$

Based on Definition $3, I=\{1\}$ and $\bar{I}=\{2\}$. Criterion 1 is not weak difference independent of criterion 2. It can be shown that the first inequality leads to $0.3>0.2$ while the second inequality is held as long as $2[\mu(1,2)-\mu(2)]<[\mu(1)-\mu(\varnothing)]$. Therefore, this preference example violating weak difference independence can be represented by unipolar capacity.

In order to investigate the ability of unipolar capacity in representing preference situations violating weak difference independence further, we consider Result 4. If $s_{1}<s_{2}$, then (for a fixed value of criterion $2, u_{2}^{j}$ ) preference difference created by increasing criterion 1 from $u_{1}^{1}$ to $u_{1}^{2}$ will be equal to or 
smaller than the difference obtained by increasing $u_{1}^{3}$ to $u_{1}^{4}$ for $u_{1}^{2}-u_{1}^{1}=u_{1}^{4}-u_{1}^{3}$ and this will be valid for all levels of criterion 2, i.e. $v\left(u_{1}^{2}, u_{2}^{j}, u_{N \backslash\{1,2\}}\right)-v\left(u_{1}^{1}, u_{2}^{j}, u_{N \backslash\{1,2\}}\right) \leq v\left(u_{1}^{4}, u_{2}^{j}, u_{N \backslash\{1,2\}}\right)-$ $v\left(u_{1}^{3}, u_{2}^{j}, u_{N \backslash\{1,2\}}\right), \forall j$. Consequently, unipolar capacity cannot represent preference situations violating weak difference independence if $x_{1}^{2}-x_{1}^{1}=x_{1}^{4}-x_{1}^{3}$ in Definition 3 (note that $u=x$ for the unipolar capacity). Fig. 5 illustrates this preference example for $s_{1}<s_{2}$.

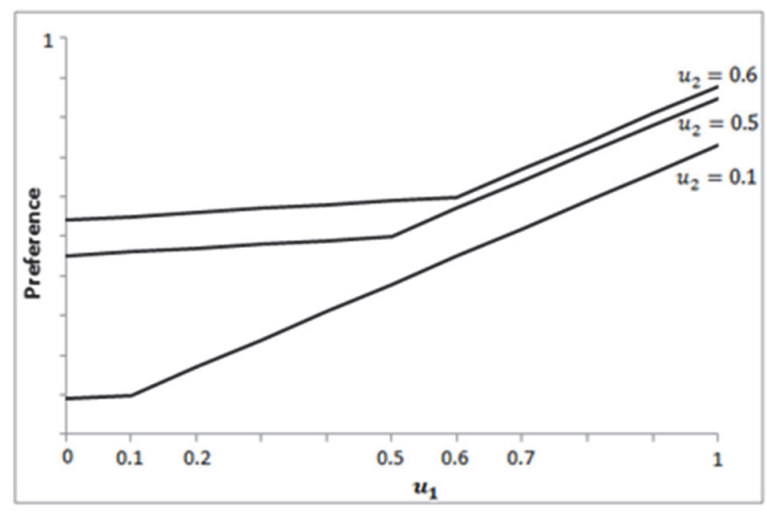

Fig. 5. Choquet integral result for the example violating weak difference independence condition.

\subsection{Interaction structures that can be modeled by k-ary capacity}

Example 1: As a motivating example, suppose that Figure 6 represents the preference structure of the DM for two criteria. The figures illustrate effect of criterion 1 on the preference for different levels of criterion 2. This type of preference structure cannot be represented by unipolar capacity due to violation of Result 3 given in Table 1. Result 3 states that preference differences should increase or decrease monotonically or should be equal. However, in Figure 6(a), $d_{2}>d_{1}$ while $d_{4}<d_{3}$.

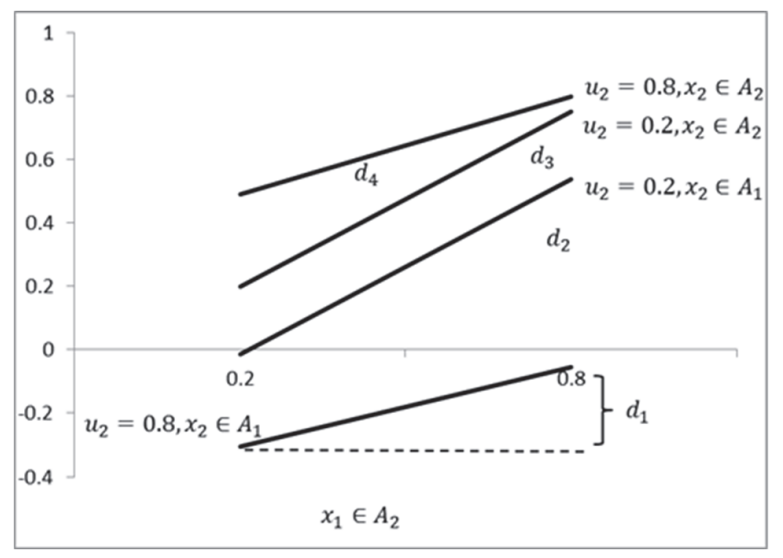

(a)

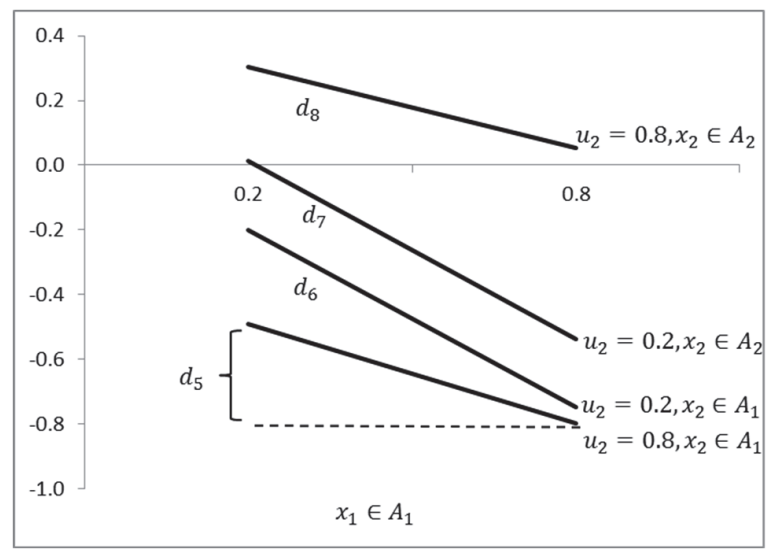

(b)

Fig. 6. Preference structure for Example 1. (a) Levels of criterion 1 belong to region $A_{2}$. (b) Levels of criterion 1 belong to region $A_{1}$.

Levels of criterion 1 are above a neutral value in Fig. 6(a), while they are below a neutral value in (b). The regions above and below the neutral value are denoted by $A_{2}$ and $A_{1}$, respectively in Fig. 6 . The two criteria are complementary when the values of criterion 2 are within region $A 1$ while the criteria are substitutive when criterion 2 is in region $A_{2}$. This preference structure can be represented by k-ary capacity. For example, 3 -ary capacity values based on reference levels 1 , 0 , and -1 (i.e., bi-capacity) given below produce preference scores in agreement with this preference structure. 
$\mu(\{1,2\}, \emptyset)=-1, \mu(\{2\},\{1\})=-0.067, \mu(\{2\}, \emptyset)=-0.48$,

$\mu(\{1\},\{2\})=-0.067, \mu(\varnothing,\{1,2\})=1, \mu(\varnothing,\{2\})=0.5$,

$\mu(\{1\}, \emptyset)=-0.92, \mu(\varnothing,\{1\})=0.92, \mu(\emptyset, \varnothing)=0$.

This example shows that different from unipolar capacity, differences in preferences are not required to change monotonically in the k-ary capacity case.

In order to investigate the effect of criterion 1 on preference (i.e., effect of changing the value of criterion 1 from $u_{1}^{1}$ to $u_{1}^{2}$ ) at different levels of criterion 2, we consider again Fig. 3 by taking $x_{1}^{1}, x_{1}^{2} \in$ $A_{m}$ and $x_{2}^{j} \in A_{n}$ and modify Eq. (11) for the k-ary case as given by Eq. (17).

$$
d_{A_{n}, j}=\left\{\begin{array}{l}
\left(u_{1}^{2}-u_{1}^{1}\right)\left[\mu_{\mathcal{A}}(1)-\mu_{\mathcal{A}}(\varnothing)\right], \text { if } u_{2}^{j} \leq u_{1}^{1}<u_{1}^{2} \\
\left(u_{2}^{j}-u_{1}^{1}\right)\left[\mu_{\mathcal{A}}(1,2)-\mu_{\mathcal{A}}(2)\right]+\left(u_{1}^{2}-u_{2}^{j}\right)\left[\mu_{\mathcal{A}}(1)-\mu_{\mathcal{A}}(\emptyset)\right], \text { if } u_{1}^{1}<u_{2}^{j}<u_{1}^{2} \\
\left(u_{1}^{2}-u_{1}^{1}\right)\left[\mu_{\mathcal{A}}(1,2)-\mu_{\mathcal{A}}(2)\right], \text { if } u_{1}^{1}<u_{1}^{2} \leq u_{2}^{j}
\end{array}\right.
$$

Let us define the operation $\Delta_{t}$ for k-ary capacity $\mu$ as follows:

$\Delta_{t}^{A_{n}^{+},[\ldots]}=\mu\left(B_{q}^{-}, \ldots, B_{1}^{-}, B_{1}^{+}, \ldots, B_{n}^{+} \cup\{t\}, \ldots, B_{p}^{+}\right)-\mu\left(B_{q}^{-}, \ldots, B_{1}^{-}, B_{1}^{+}, \ldots, B_{n-1}^{+} \cup\{t\}, B_{n}^{+}, \ldots, B_{p}^{+}\right)$,
$\Delta_{t}^{A_{1}^{+},[\ldots]}=\mu\left(B_{q}^{-}, \ldots, B_{1}^{-}, B_{1}^{+} \cup\{t\}, \ldots, B_{p}^{+}\right)-\mu\left(B_{q}^{-}, \ldots, B_{1}^{-}, B_{1}^{+}, \ldots, B_{p}^{+}\right)$,
$\Delta_{t}^{A_{n}^{-},[\ldots]}=\mu\left(B_{q}^{-}, \ldots, B_{n}^{-} \cup\{t\}, \ldots, B_{1}^{-}, B_{1}^{+}, \ldots, B_{p}^{+}\right)-\mu\left(B_{q}^{-}, \ldots, B_{n}^{-}, B_{n-1}^{-} \cup\{t\}, \ldots, B_{1}^{-}, B_{1}^{+}, \ldots, B_{p}^{+}\right)$,
$\Delta_{t}^{A_{1}^{-},[\ldots]}=\mu\left(B_{q}^{-}, \ldots, B_{1}^{-} \cup\{t\}, B_{1}^{+}, \ldots, B_{p}^{+}\right)-\mu\left(B_{q}^{-}, \ldots, B_{1}^{-}, B_{1}^{+}, \ldots, B_{p}^{+}\right)$.

where,

$B_{q}^{-}=\left\{i \mid i \neq t, i \in A_{q}^{-}, u_{i} \geq u_{t}\right\}$,

$B_{q-1}^{-}=\left\{i \mid i \neq t, i \in A_{q-1}^{-}, u_{i} \geq u_{t}\right\} \cup\left\{i \mid i \in A_{q}^{-}, u_{i}<u_{t}\right\}$

$\vdots$

$B_{1}^{-}=\left\{i \mid i \neq t, i \in A_{1}^{-}, u_{i} \geq u_{t}\right\} \cup\left\{i \mid i \in A_{2}^{-}, u_{i}<u_{t}\right\}$,

$B_{1}^{+}=\left\{i \mid i \neq t, i \in A_{1}^{+}, u_{i} \geq u_{t}\right\} \cup\left\{i \mid i \in A_{2}^{+}, u_{i}<u_{t}\right\}$,

$\vdots$

$B_{p-1}^{+}=\left\{i \mid i \neq t, i \in A_{p-1}^{+}, u_{i} \geq u_{t}\right\} \cup\left\{i \mid i \in A_{p}^{+}, u_{i}<u_{t}\right\}$,

$B_{p}^{+}=\left\{i \mid i \neq t, i \in A_{p}^{+}, u_{i} \geq u_{t}\right\}$,

$k=q+p+1$.

$\Delta_{t}^{T,\left[A_{n},+\right]}$ denotes that $t \in T, i \in A_{n}$ and $u_{i} \geq u_{t}$, where $T \in\left\{A_{q}^{-}, \ldots, A_{1}^{-}, A_{1}^{+}, \ldots, A_{p}^{+}\right\}$. Similarly, the operation is shown by $\Delta_{t}^{T,\left[A_{n},-\right]}$ if $t \in T, i \in A_{n}$ and $u_{i}<u_{t}$.

Then, the preference difference of the two alternatives $\left(u_{1}^{2}, u_{2}^{j}, 0_{N \backslash\{1,2\}}\right)$ and $\left(u_{1}^{1}, u_{2}^{j}, 0_{N \backslash\{1,2\}}\right)$, (where $x_{2}^{j} \in A_{n}$ and $\left.x_{1}^{1}, x_{1}^{2} \in A_{m}\right)$ denoted by $d_{A_{n}, j}$, is given by Eq. (22). 


$$
d_{A_{n}, j}=\left\{\begin{array}{l}
\left(u_{1}^{2}-u_{1}^{1}\right) \Delta_{1}^{A_{m},\left[A_{n},-\right]} \text { if } u_{2}^{j} \leq u_{1}^{1}<u_{1}^{2} \\
\left(u_{1}^{2}-u_{2}^{j}\right) \Delta_{1}^{A_{m},\left[A_{n},-\right]}+\left(u_{2}^{j}-u_{1}^{1}\right) \Delta_{1}^{A_{m},\left[A_{n},+\right]} \text { if } u_{1}^{1}<u_{2}^{j}<u_{1}^{2} \\
\left(u_{1}^{2}-u_{1}^{1}\right) \Delta_{1}^{A_{m},\left[A_{n},+\right]} \text { if } u_{1}^{1}<u_{1}^{2} \leq u_{2}^{j}
\end{array}\right.
$$

It can be seen from Eqs. (18-21) that $\Delta_{1}^{A_{m},\left[A_{n}^{+},+\right]}=\Delta_{1}^{A_{m},\left[A_{n+1}^{+},-\right]}, \quad \Delta_{1}^{A_{m},\left[A_{n-1}^{-},+\right]}=\Delta_{1}^{A_{m},\left[A_{n}^{-},-\right]}$and $\Delta_{1}^{A_{m},\left[A_{1}^{-},-\right]}=\Delta_{1}^{A_{m},\left[A_{1}^{+},-\right]}$. Thus, preference differences of alternatives (1) and (2) will be equal to each other for some conditions. For example, $d_{A_{n}^{+}, j}=d_{A_{n+1}^{+}, j+1}$ for $u_{2}^{j} \geq u_{1}^{2}>u_{1}^{1} \geq u_{2}^{j+1}$, since $d_{A_{n}^{+}, j}=$ $\left(u_{1}^{2}-u_{1}^{1}\right) \Delta_{1}^{A_{m},\left[A_{n}^{+},+\right]}$and $d_{A_{n+1}^{+}, j+1}=\left(u_{1}^{2}-u_{1}^{1}\right) \Delta_{1}^{A_{m},}\left[A_{n+1}^{+},-\right]$for $u_{2}^{j} \geq u_{1}^{2}>u_{1}^{1} \geq u_{2}^{j+1}$.

Based on Eq. (22), conditions required to represent change in preference differences for two criteria by k-ary capacity are shown in Table 2 .

Fig. 6(a) imposes the following inequalities which represent a preference structure violating weak difference independence. Bi-capacity values that satisfy these conditions are given in Eq. (16). Note that bi-capacity is able to represent this preference structure violating the weak difference independence condition although $\left|u_{2}^{2}-u_{2}^{1}\right|=\left|u_{2}^{4}-u_{2}^{3}\right|$.

$$
\begin{aligned}
& v(0.2,0.8)-v(0.2,0.2)>v(0.2,-0.2)-v(0.2,-0.8) \\
& v(0.8,0.8)-v(0.8,0.2)<v(0.8,-0.2)-v(0.8,-0.8)
\end{aligned}
$$

\section{Table 2}

Conditions required to model change in preference difference by k-ary capacity

Plot of preference differences

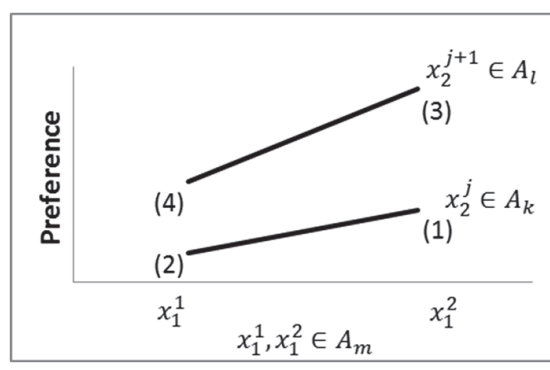

Result 5. If $A_{k}=A_{l}$ conditions of unipolar capacity are valid.

Result 6. If $A_{k} \neq A_{l}$ the two lines are parallel for the following cases:

$$
\begin{aligned}
& -A_{k}=A_{n}^{+}, A_{l}=A_{n+1}^{+} \text {and } u_{2}^{j} \geq u_{1}^{2}>u_{1}^{1} \geq u_{2}^{j+1} \\
& -A_{k}=A_{n+1}^{-}, A_{l}=A_{n}^{-} \text {and } u_{2}^{j+1} \geq u_{1}^{2}>u_{1}^{1} \geq u_{2}^{j} \\
& -A_{k}=A_{1}^{-}, A_{l}=A_{1}^{+} \text {and } u_{2}^{j} \leq u_{1}^{1}<u_{1}^{2}, u_{2}^{j+1} \leq u_{1}^{1}<u_{1}^{2}
\end{aligned}
$$

Result 7. For different regions ( such as $A_{k} \neq A_{l} \neq A_{n}$ ), preference differences do not need to behave monotonically as long as the conditions given in the Result 6 are satisfied.

\section{A framework for analyzing interactions}

In this section, results of Section 4 are used to design a framework to analyze interactions. This framework consists of three steps as described in the subsequent paragraphs. 
Step 1: Information about criteria of the problem and their important properties such as their units, ranges, targets, possible lower and upper acceptable limits are gathered. Levels for continuous type criteria are specified to facilitate the construction of the interaction plots. When determining these levels it is important to consider some decision examples. DM may have different preference structures at different intervals of criteria evaluations. For example, in most of the quality problems quality characteristic values have levels as acceptable, rework and scrap. When one of the quality characteristics of the item under consideration has a scrap value, the item may be scrapped regardless of values of the other quality characteristics. In this case, the effect of deviation of one quality characteristic from its target on preference is different at different intervals of the value gained by another quality characteristic. A practitioner from manufacturing environment provides the following example: In the sole attachment process of shoe manufacturing, the sole is attached to upper part of the shoe. A cut in the leather can occur during this process. Another characteristic that is controlled is back height. When a cut occurs or back height is longer than target, the sole can be reattached to the upper part from an upper position. Since this operation causes back height to become shorter, the effect of cut is different for three levels of back height, namely target, long and short. Similarly, a criterion having a value deemed as risky may necessitate careful attention on the other criteria in which case allowable limits for them are tightened. In this case, again, effect of a criterion on preference depends on the level of another criterion.

Step 2: In the second step, it is explored with the DM whether or not there are interacting criteria. The analyst draws interaction plots for the criteria based on the information obtained in Step 1. For each level of one criterion, the analyst asks the DM to indicate the effect of increase in the value of another criterion. If there are more than two criteria, then the interaction plots are constructed by holding the levels of other criteria at reasonable values.

Constructing each of these plots necessitates pairwise comparisons of at least four alternatives (see Figure 2). Most simply, these alternatives can be created by taking the least and most desirable levels of the criteria under consideration (while the other criteria are at their fixed levels).

Step 3: The main aim of this study is to provide guidelines to determine the appropriate form of the aggregation function in practical applications by concentrating on the Choquet integral. Accordingly, once the interaction plots are constructed an appropriate aggregation method is chosen in the third step based on the type of the decision making problem and the revealed interaction structure.

If the problem is ranking the alternatives and the preference orientation does not change, i.e. preference independence condition is satisfied, then there are many methods that can be used such as ELECTRE, PROMETHEE, ordinal additive value function of MAVT. If we have a ranking problem, but differences between the alternatives are also important and there is no dependency between the criteria, then Analytic Hierarchy Process (AHP) or a measurable additive value function of MAVT can be used. If the lines of the interaction plot cannot be interpreted as parallel but the order of the change in the preferences remains same, i.e. weak difference independence hold, then a multiplicative value function of MAVT can be used. If these conditions are not satisfied, there are a limited number of methods that can be used. In this study, we concentrate on Choquet integral. ANP is another method that can handle interactions, which needs a similar study to this one to clarify the interaction types it can handle. Table 1 presents decision structures that can be modeled by Choquet integral with respect to unipolar capacity and Table 2 shows decision structures that can be modeled by Choquet integral with respect to k-ary capacity. Therefore, the appropriate capacity type can be determined by comparing the interaction plots constructed in Step 2 with the results presented in these tables.

\section{An illustrative example from manufacturing environment}

In this section, we apply the framework introduced in Section 5 to a real life example which was studied in the course of a project related with constructing value functions to determine preference scores for decision alternatives in quality control and improvement activities under preferential dependence. 
Step 1: Two quality characteristics of interest at a turning operation are thickness and surface roughness where thickness is the distance between outer and inner diameters. Thickness is denoted by 1 and the surface roughness is denoted by 2 . The quality characteristic values are classified as acceptable, rework and scrap. Scrap values are shown by S, while R and A are used for rework and acceptable values, respectively. Decision structures are different at these different intervals of criteria evaluations. When one of the quality characteristics has a scrap value, then the item is scrapped regardless of the value of the other one and these scrapped items are not preference ordered. Thus, both of the quality characteristics have (conditional) veto effect. If a rework operation is performed for the thickness, then surface roughness will also have a new value. Therefore, the value of the surface roughness plays no role on the preference score when the thickness needs to be reworked.

Step 2: Interaction plots are constructed based on the above explanations. When surface roughness has a value within the specification limits that define the acceptable region for the quality characteristic, preference structure at different levels of thickness can be represented by Fig. 7(a). As can be seen from the figure, when thickness value is within the scrap region or rework region, surface roughness has no effect on the preference. Preference structure is similar to that of Fig. 7(a) if surface roughness is within the rework limits. Fig. 7(b) illustrates the preference structure for thickness within specification limits (i.e. in acceptable region).
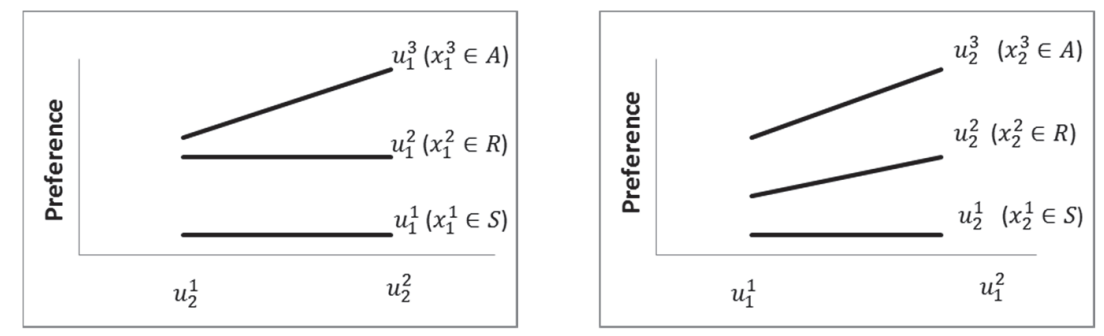

(a)

(b)

Fig. 7. Preference structure for the illustrative example. (a) Effect of surface roughness for different values of thickness. (b) Effect of thickness for different values of surface roughness

Step 3: Interaction plots constructed in Step 2 will be used in Step 3 to determine the appropriate capacity type using Tables 1 and 2 (i.e. the appropriate capacity type will be determined by comparing the interaction plots constructed in Step 2 with the results presented in Tables 1 and 2).

Consider first the preference structure illustrated by Figure 7(a). According to Table 1, since the lines for $u_{1}^{1}$ and $u_{1}^{2}$ are parallel to each other and the line for $u_{1}^{3}$ is not parallel to them, $u_{1}^{1}$ and $u_{1}^{2}$ should be on the same side of $u_{2}^{1}$ and $u_{2}^{2}$ in the ranking of the criteria values while $u_{1}^{3}$ should have a different position.

Considering the above explanation together with the information that $x_{1}^{1}$ is a scrap, $x_{1}^{2}$ is a rework value and $x_{2}^{1}, x_{2}^{2}$ and $x_{1}^{3}$ are acceptable values, one of the following two rankings is valid $(u=x$, since we consider the unipolar capacity):

$$
\begin{aligned}
& u_{1}^{1}<u_{1}^{2} \leq u_{2}^{1}<u_{2}^{2} \leq u_{1}^{3} \\
& u_{1}^{1}<u_{1}^{2} \leq u_{2}^{1}<u_{1}^{3} \leq u_{2}^{2}
\end{aligned}
$$

When the ranking of the quality characteristic values is as shown by (25), the relation for preference differences as $d_{1}=d_{2}<d_{3}\left(d_{1}\right.$ denotes the preference difference between two alternatives related with the scrap line in Figure 7(a), and so on) is in line with Results 1 and 3 of Table 1 (Result 1: $u_{1}^{1}$ and $u_{1}^{2}$ are smaller than $u_{2}^{1}$, therefore $d_{1}=d_{2}$; Result 3: $u_{1}^{3}$ has a different position than these two values $\left(u_{1}^{1}\right.$ and $\left.u_{1}^{2}\right)$ regarding the values of criterion 2 , therefore $d_{3}$ can be smaller or larger than $d_{1}$ and $d_{2}$ ). 
However, this means that the preference structure illustrated by Fig. 7(a) can be represented only for acceptable values of surface roughness smaller than acceptable values of thickness.

For the ranking shown by (26), we know from Result 4 of Table 1 that the preference structure can be represented partially as illustrated by Figure 8. As explained in Section 4 (Result 4 in Table 1), preference function for surface roughness for each level of thickness will be a piecewise linear function with breakpoint occurring at the value of thickness. The lines for $u_{1}^{1}$ and $u_{1}^{2}$ in Fig. 7(a) are associated with the second segments of the functions since both of these values are smaller than $u_{2}^{1}$ and $u_{2}^{2}$, hence $s_{2}=0$. Since $u_{2}^{1}<u_{1}^{3}<u_{2}^{2}$ in Eq. (26) and $s_{2}=0$, when the value of the thickness is $u_{1}^{3}$, surface roughness will have no effect for the values within the interval $\left(u_{1}^{3}, u_{2}^{2}\right]$ while it will have some effect on the preference for the $\left[u_{2}^{1}, u_{1}^{3}\right]$ part of the interval $\left[u_{2}^{1}, u_{2}^{2}\right]$ as shown in Figure 8 .

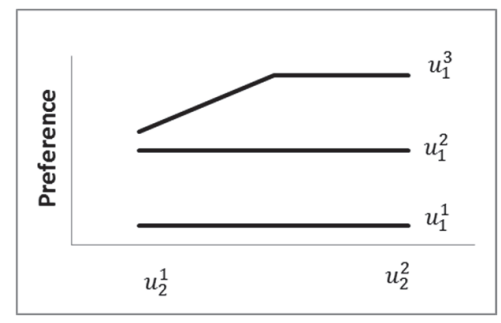

Fig. 8. Partial representation of the preference structure shown by Fig. 7(a)

Consequently, it is seen that the preference structure illustrated by Fig. 7(a) cannot be represented by the unipolar capacity for all possible values of the quality characteristics.

In Fig. 7(b), $x_{1}^{1}, x_{1}^{2} \in \mathrm{A}, x_{2}^{1} \in \mathrm{S}$ and $x_{2}^{2} \in \mathrm{R}$. Therefore, $u_{2}^{1}<u_{2}^{2}<u_{1}^{1}<u_{1}^{2}$ if the unipolar capacity will be used. Result 1 of Table 1 states that scrap and rework lines should be parallel for the present ranking. Consequently, the preference structure represented by Figure 7(b) cannot be represented by the unipolar capacity.

Now, we try to model the preference structure by k-ary capacity by considering 3 regions as scrap, rework and acceptable. Scrap and rework regions are taken as negative while the acceptable region is taken as positive (i.e, we consider 4 -ary capacity with reference levels $-1, \alpha^{-1}, 0$, and 1 ; therefore, $i \in S$ if $x_{i} \in\left[-1, \alpha^{-1}\right), i \in R$ if $x_{i} \in\left[\alpha^{-1}, 0\right)$, and $i \in A$ if $\left.x_{i} \in(0,1], i=1,2\right)$. Since scrapped items are not preference ordered, all of the scrap values can be assigned -1 (i.e. if the value of a quality characteristics is in the scrap region, then the corresponding $x$ value for this quality characteristic is -1 and based on Equation (10) the $u$ value is 1).

By assigning -1 to all of the capacities having a quality characteristic in the scrap region $(\mu(\{1,2\}, \emptyset, \emptyset)=-1, \mu(\{1\},\{2\}, \emptyset)=-1, \mu(\{1\}, \emptyset,\{2\})=-1, \mu(\{1\}, \emptyset, \emptyset)=-1, \mu(\{2\},\{1\}, \emptyset)=$ $-1, \mu(\{2\}, \emptyset,\{1\})=-1, \mu(\{2\}, \emptyset, \emptyset)=-1)$, the veto effect of the quality characteristics can be represented.

Considering the Fig. 7(b), scrap and rework lines do not need to be parallel when using the 4-ary capacity: Considering these two lines in terms of Result 6 of Table $2, A_{k}=A_{2}^{-}$(scrap line) and $A_{l}=$ $A_{1}^{-}$(rework line). Hence, we need to check the second condition given in Result 6 to understand whether these two lines should be parallel or not. Since $u_{1}^{1}<u_{1}^{2} \leq u_{2}^{1}=1$ and $u_{2}^{2} \leq u_{2}^{1}$, the second condition given in Result 6 does not incur and hence they do not need to be parallel. When it comes to accept and rework lines, $A_{k}=A_{1}^{-}$(rework line) and $A_{l}=A_{1}^{+}$(accept line). Based on the third condition of Result 6 , the two lines should be parallel for $u_{2}^{j} \leq u_{1}^{1}<u_{1}^{2}, u_{2}^{j+1} \leq u_{1}^{1}<u_{1}^{2}$. Accordingly, the preference structure that can be modeled by Choquet integral with respect to 4-ary capacity is illustrated in Figure 9. 


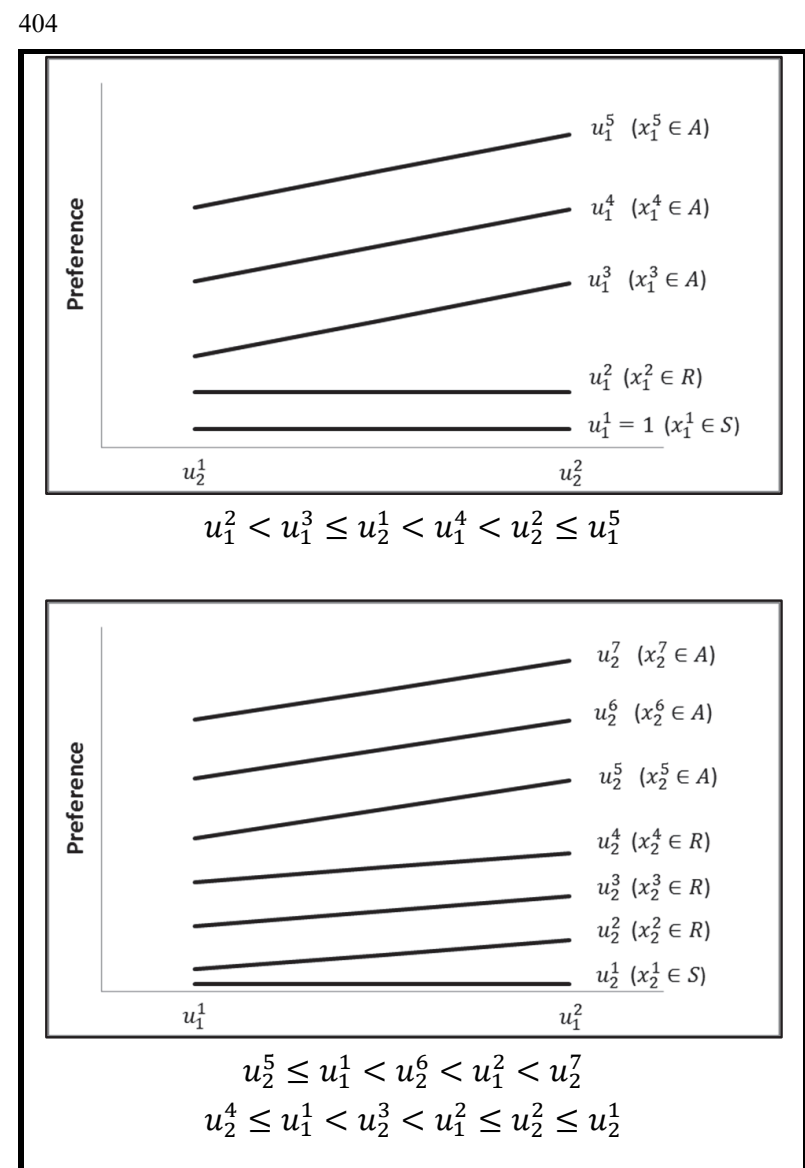

(a)

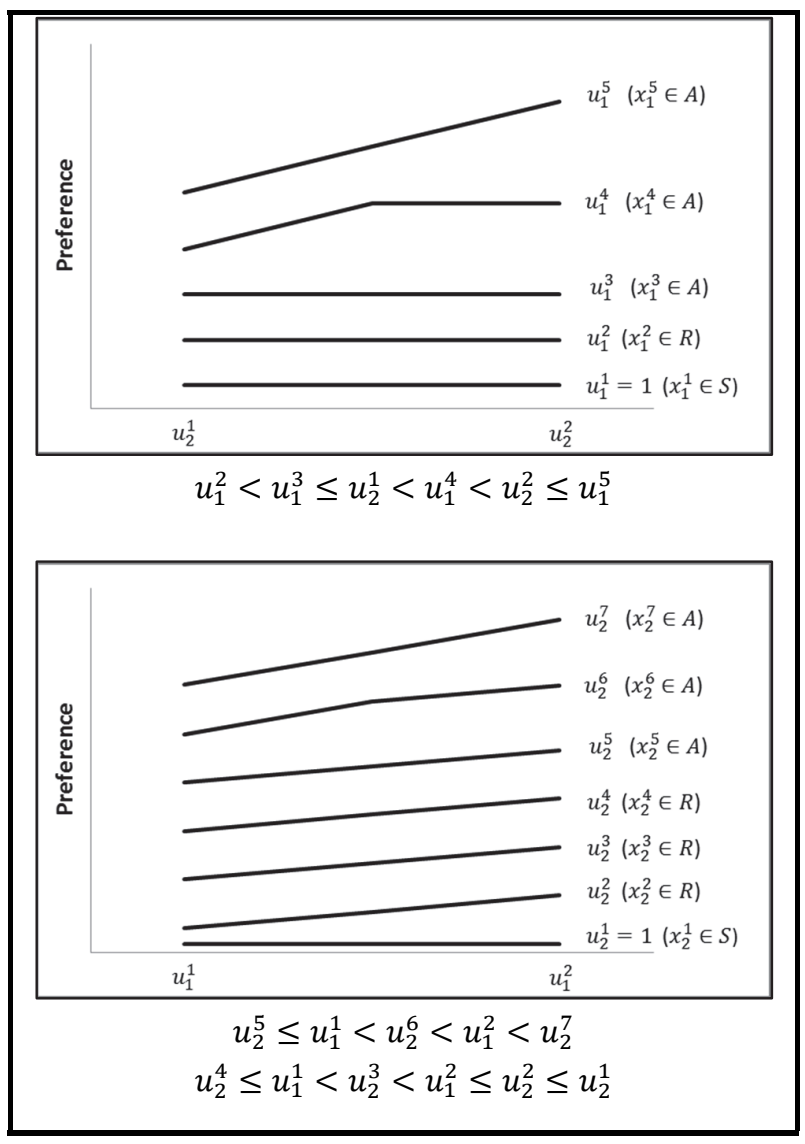

(b)

Fig. 9. (a) Preference structure of the DM as illustrated by Fig. 7. (b) Preference structure that can be modeled by the Choquet integral with respect to the 4 -ary capacity

\section{Discussion and conclusion}

The aim of this study was to examine in detail the preference structures which could be modeled by Choquet integral. It has been shown that under certain conditions, Choquet integral with respect unipolar and k-ary capacities could be used to model preferences although preference independence or weak difference independence assumptions of MAVT were not satisfied. This study also has presented applications of the k-ary capacity which has only been studied in the literature theoretically to the best of our knowledge. It should be noted that Choquet integral reduces to a weighted sum when the criteria are independent and produces a linear preference function. One can see for example, multivariate loss functions (Ko et al., 2005) to construct a quadratic preference function. In the case of interaction, Choquet integral produces a piecewise linear preference function. The results of the investigation of interaction types that can be modeled by the unipolar and k-ary capacities were used to provide guidelines for specifying the appropriate capacity type in practical applications. The guidelines have been presented in Tables 1 and 2. Interaction plots constructed by following the instructions provided in Section 5 are compared with the results presented in these tables to decide on the appropriate capacity type. These guidelines can be combined with previously proposed approaches for specification of capacity values (for example Sicilia et al. (2003)) and can be used as the first step of such approaches. Moreover, preference information elicited to construct the interaction plots can be used in subsequent steps of those approaches. Since most of these approaches are proposed to specify unipolar capacities, they can be extended to consider k-ary capacities.

In order to explain the above paragraph more clearly, we use Fig. 10. Constructing a value function, i.e. specification of the parameters of a value function (specification of capacity values in our case) 
from decision examples is called preference disaggregation (Doumpos \& Zopounidis, 2011). Framework of the preference disaggregation approach is presented in Fig. 10. In the first step, the general form of the value function should be determined (weighted sum, Choquet integral, and so on). Then, DM is asked to provide preference information on some reference alternatives. This information is used to determine the parameters of the value function usually by constructing and solving an optimization problem, i.e. an optimization problem is constructed and solved to find the parameters so that the resulting value function is as consistent as possible with the preference information provided by the DM. Afterwards, these parameters are adjusted interactively with the DM: Some new evaluations are generated by the resulting function and presented to the DM. If these evaluations are in accordance with the DM preferences then the value function can be applied to new decision instances. Otherwise, the information obtained at this step is used to adjust the parameters through solving the optimization problem modified with new constraints. Accordingly, the current study constitutes the shaded steps in Figure 10. The provided guidelines are used to determine the appropriate form of the value function and the preference evaluations obtained to construct the interaction plots can be used to construct the optimization problem to find the parameter values (i.e. capacity values).

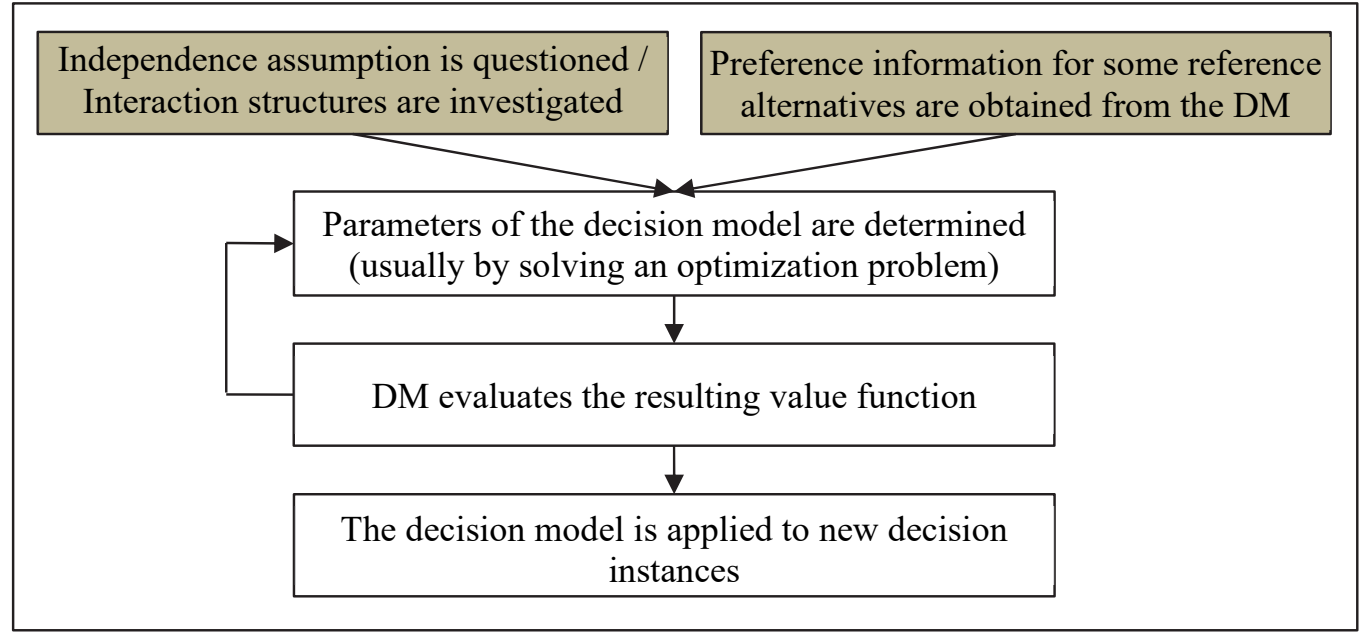

Fig. 10. Framework of the preference disaggregation approach.

Throughout the study, existence of a common scale for criteria evaluations was assumed. Therefore, integrating construction of such a common scale to the study is considered as a future development.

\section{Acknowledgement}

This study was supported by Scientific Research Projects Committee of Eskisehir Osmangazi University (Project No: 201215016).

\section{References}

Abdullah, L., Zulkifli, N., Liao, H., Herrera-Viedma, E., \& Al-Barakati, A. (2019). An interval-valued intuitionistic fuzzy DEMATEL method combined with Choquet integral for sustainable solid waste management. Engineering Applications of Artificial Intelligence, 82, 207-215.

Angilella, S., Corrente, S., \& Greco, S. (2015). Stochastic multiobjective acceptability analysis for the Choquet integral preference model and the scale construction problem. European Journal of Operational Research, 240, 172-182.

Angilella, S., Corrente, S., Greco, S., \& Słowiński, R. (2013, March). Multiple criteria hierarchy process for the Choquet integral. In Proceedings of International Conference on Evolutionary MultiCriterion Optimization (pp. 475-489). Springer, Berlin, Heidelberg.

Angilella, S., Greco, S., Lamantia, F., \& Matarazzo B. (2004). Assessing non-additive utility for multicriteria decision aid. European Journal of Operational Research, 158(3), 734-744. 
Angilella, S., Greco, S., \& Matarazzo, B. (2010). Non-additive robust ordinal regression: A multiple criteria decision model based on the Choquet integral. European Journal of Operational Research, 201(1), 277-288.

Bottero, M., Ferretti, V., Figueira, J.R., Greco, S., \& Roy, B. (2018). On the Choquet multiple criteria preference aggregation model: Theoretical and practical insights from a real-world application. European Journal of Operational Research, 271(1), 120-140.

Choquet, G. (1953). Theory of capacities. Annales de l'Institut Fourier, 5, 131-295.

Corrente, S., Figueira, J.R., \& Greco, S. (2014). Dealing with interaction between bipolar multiple criteria preferences in PROMETHEE methods. Annals of Operations Research, 217(1), 137-164.

Doumpos, M., \& Zopounidis, C. (2011). Preference disaggregation and statistical learning for multicriteria decision support: A review. European Journal of Operational Research, 209, 203-214.

Dyer, J. S. (2005). MAUT - multiattribute utility theory. In Figueira, J.R., Greco, S., Ehrgott, M. (Eds.), Multiple criteria decision analysis: state of the art surveys (pp. 265-295). Springer, New York, NY.

Figueira, J.R., Greco, S. \& Roy, B. (2009). ELECTRE methods with interaction between criteria: An extension of the concordance index. European Journal of Operational Research, 199(2), 478-495.

Grabisch, M. (2004, July). The Choquet integral as a linear interpolator. In Proceedings of 10th Int. Conf. on Information Processing and Management of Uncertainty in Knowledge-Based Systems (IPMU 2004) (pp. 373-378).

Grabisch, M., Kojadinovic, I., \& Meyer, P. (2008). A review of methods for capacity identification in Choquet integral based multi-attribute utility theory: Applications of the Kappalab R package. European Journal of Operational Research, 186(2), 766-785.

Grabisch, M., \& Labreuche, C. (2003, September). Capacities on lattices and k-ary capacities. In Proceedings of EUSFLAT Conf. (pp. 304-307). Zittau, Germany.

Grabisch, M., \& Labreuche, C. (2005a). Fuzzy measures and integrals in MCDA. In Figueira, J.R., Greco, S., Ehrgott, M. (Eds.), Multiple criteria decision analysis: state of the art surveys (pp. 563604). Springer, New York, NY.

Grabisch, M., \& Labreuche, C. (2005b). Bi-capacities-I: definition, Möbius transform and interaction. Fuzzy Sets and Systems, 151, 211-236.

Grabisch, M., \& Labreuche, C. (2005c). Bi-capacities-II: the Choquet integral. Fuzzy Sets and Systems, 151, 237-259.

Grabisch, M., \& Labreuche, C. (2008a). A decade of application of the Choquet and Sugeno integrals in multi-criteria decision aid. 4OR, 6(1), 1-44.

Grabisch, M., \& Labreuche, C. (2008b). Bipolarization of posets and natural interpolation. Journal of Mathematical Analysis and Applications, 343, 1080-1097.

Gurbuz, T., \& Albayrak, Y.E. (2014). An engineering approach to human resources performance evaluation: Hybrid MCDM application with interactions. Applied Soft Computing, 21, 365-375.

Gurbuz, T., Alptekin, S.E., \& Isiklar Alptekin, G. (2012). A hybrid MCDM methodology for ERP selection problem with interacting criteria. Decision Support Systems, 54, 206-214.

Ko, Y.H., Kim, K.J., \& Jun, C.H. (2005) A new loss function-based method for multiresponse optimization. Journal of Quality Technology, 37(1), 50-59.

Labreuche, C., \& Grabisch, M. (2007). The representation of conditional importance between criteria. Annals of Operations Research, 154, 93-122.

Lovász, L. (1983). Submodular functions and convexity. In Bachem, A., Grötschel, M., Korte, B. (Eds.), Mathematical Programming: The State of the Art (pp. 235-257). Springer, Berlin, Heidelberg.

Marichal, J.L. (2002). Aggregation of interacting criteria by means of the discrete Choquet integral. In Calvo, T., Mayor, G., Mesiar, R. (Eds.), Aggregation operators: New Trends and Applications (pp. 224-244). Physica, Heidelberg.

Marichal, J.L., \& Roubens, M. (2000). Determination of weights of interacting criteria from a reference set. European Journal of Operational Research, 124(3), 641-650. 
Ming-Lang, T., Chiang, J.H., \& Lan, L.W. (2009). Selection of optimal supplier in supply chain management strategy with analytic network process and Choquet integral. Computers \& Industrial Engineering, 57, 330-340.

Montgomery, D.C. (2005). Design and Analysis of Experiments. John Wiley and Sons, Inc.

Sicilia, M.Á., Barriocanal, E.G., \& Calvo, T. (2003). An inquiry-based method for Choquet integralbased aggregation of interface usability parameters. Kybernetika, 39(5), 601-614.

Sugeno, M. (1974). Theory of fuzzy integrals and its applications. Doct. Thesis, Tokyo Institute of technology.

Timonin, M. (2013). Robust optimization of the Choquet integral. Fuzzy Sets and Systems, 213, 27-46. Wang, L., Wang, H., Xu, Z., \& Ren, Z. (2019). The interval-valued hesitant Pythagorean fuzzy set and its applications with extended TOPSIS and Choquet integral-based method. International Journal of Intelligent Systems, 34(6), 1063-1085.

\section{Appendix A. An example for application of Eq. (7)}

For illustrating application of Eq. (7) let us consider the alternative $\boldsymbol{x}=(-0.2,0.8,-0.3)$. Here, $N^{+}=$ $\{2\}$ and $N^{-}=\{1,3\}$. Then,

$$
\begin{aligned}
B C_{v}(-0.2,0.8, & -0.3)=C_{\mu_{N^{+}, N^{-}}}(0.2,0.8,0.3) \\
& =0.2\left[\mu_{N^{+}, N^{-}}(\{1,2,3\})-\mu_{N^{+}, N^{-}}(\{2,3\})\right]+0.3\left[\mu_{N^{+}, N^{-}}(\{2,3\})-\mu_{N^{+}, N^{-}}(\{2\})\right] \\
& +0.8\left[\mu_{N^{+}, N^{-}}(\{2\})-\mu_{N^{+}, N^{-}}(\varnothing)\right] \\
& =0.2 \mu_{N^{+}, N^{-}}(\{1,2,3\})+0.1 \mu_{N^{+}, N^{-}}(\{2,3\})+0.5 \mu_{N^{+}, N^{-}}(\{2\})-0.8 \mu_{N^{+}, N^{-}}(\varnothing) \\
& =0.2 v(\{2\},\{1,3\})+0.1 v(\{2\},\{3\})+0.5 v(\{2\}, \emptyset)-0.8 v(\varnothing, \varnothing) \\
& =0.2 v(\{2\},\{1,3\})+0.1 v(\{2\},\{3\})+0.5 v(\{2\}, \emptyset)
\end{aligned}
$$

Note that $\mu_{N^{+}, N^{-}}(\{1,2,3\})=v(\{1,2,3\} \cap\{2\},\{1,2,3\} \cap\{1,3\})=v(\{2\},\{1,3\}), \quad \mu_{N^{+}, N^{-}}(\{2,3\})=$ $v(\{2,3\} \cap\{2\},\{2,3\} \cap\{1,3\})=v(\{2\},\{3\})$ and $\mu_{N^{+}, N^{-}}(\{2\})=v(\{2\} \cap\{2\},\{2\} \cap\{1,3\})=v(\{2\}, \emptyset)$.

\section{Appendix B. An example for application of the formulas related with the Choquet integral with respect to k-ary capacity}

Let us consider a decision situation in which alternatives are evaluated under three criteria and 5-ary capacity is used to model preferences with reference values $-1,-0.5,0,0.5$ and 1 . Then, the Choquet integral formulation for alternative $\boldsymbol{x}=(-0.85,0.65,0.4)$ is obtained as follows:

$1 \in A_{2}^{-}$since $-0.85 \in\left[\alpha^{-2}, \alpha^{-1}\right)=[-1,-0.5), \Phi_{1}(\boldsymbol{x})=\frac{\left|-0.85-\alpha^{-(2-1)}\right|}{\alpha^{-(2-1)}-\alpha^{-2}}=\frac{|-0.85-(-0.5)|}{-0.5-(-1)}=0.7$;

$2 \in A_{2}^{+}$since $0.65 \in\left(\alpha^{1}, \alpha^{2}\right]=(0.5,1], \Phi_{2}(x)=\frac{0.65-\alpha^{2-1}}{\alpha^{2}-\alpha^{2-1}}=\frac{0.65-0.5}{1-0.5}=0.3 ;$

$3 \in A_{1}^{+}$since $0.4 \in\left(\alpha^{0}, \alpha^{1}\right]=(0,0.5], \Phi_{3}(x)=\frac{0.4-\alpha^{1-1}}{\alpha^{1}-\alpha^{1-1}}=\frac{0.4-0}{0.5-0}=0.8$.

Consequently, $\Phi(\boldsymbol{x})=(0.7,0.3,0.8)$ and $A_{2}^{+}=\{2\}, A_{1}^{+}=\{3\}, A_{1}^{-}=\emptyset, A_{2}^{-}=\{1\}$.

$$
\begin{aligned}
K C_{\mu}(-0.85,0.65,0.4)=C_{\mu_{\mathcal{A}}}(0.7,0.3,0.8) & \\
& =\mu_{\mathcal{A}}(\emptyset)+0.3\left[\mu_{\mathcal{A}}(\{1,2,3\})-\mu_{\mathcal{A}}(\{1,3\})\right]+0.7\left[\mu_{\mathcal{A}}(\{1,3\})-\mu_{\mathcal{A}}(\{3\})\right] \\
& +0.8\left[\mu_{\mathcal{A}}(\{3\})-\mu_{\mathcal{A}}(\varnothing)\right] \\
& =0.2 \mu_{\mathcal{A}}(\varnothing)+0.3 \mu_{\mathcal{A}}(\{1,2,3\})+0.4 \mu_{\mathcal{A}}(\{1,3\})+0.1 \mu_{\mathcal{A}}(\{3\}) \\
=0.2 \mu(\emptyset,\{1\},\{2\}, \emptyset)+ & 0.3 \mu(\{1\}, \emptyset,\{3\},\{2\})+0.4 \mu(\{1\}, \emptyset,\{2,3\}, \emptyset)+0.1 \mu(\emptyset,\{1\},\{2,3\}, \emptyset)
\end{aligned}
$$


Note that,

$$
\begin{aligned}
& \mu_{\mathcal{A}}(\{1,3\})=\mu\left(\left(A_{2}^{-} \cap\{1,3\}\right) \cup\left(A_{3}^{-} \backslash\{1,3\}\right),\left(A_{1}^{-} \cap\{1,3\}\right) \cup\left(A_{2}^{-} \backslash\{1,3\}\right),\left(A_{1}^{+} \cap\{1,3\}\right)\right. \\
& \left.\cup\left(A_{2}^{+} \backslash\{1,3\}\right),\left(A_{2}^{+} \cap\{1,3\}\right) \cup\left(A_{3}^{+} \backslash\{1,3\}\right)\right) \\
& =\mu((\{1\} \cap\{1,3\}) \cup(\varnothing \backslash\{1,3\}),(\varnothing \cap\{1,3\}) \cup(\{1\} \backslash\{1,3\}),(\{3\} \cap\{1,3\}) \cup(\{2\} \backslash \\
& \{1,3\}),(\{2\} \cap\{1,3\}) \cup(\varnothing \backslash\{1,3\}))=\mu(\{1\}, \varnothing,\{2,3\}, \varnothing) \\
& \mu_{\mathcal{A}}(\{3\})=\mu\left(\left(A_{2}^{-} \cap\{3\}\right) \cup\left(A_{3}^{-} \backslash\{3\}\right),\left(A_{1}^{-} \cap\{3\}\right) \cup\left(A_{2}^{-} \backslash\{3\}\right),\left(A_{1}^{+} \cap\{3\}\right)\right. \\
& \left.\cup\left(A_{2}^{+} \backslash\{3\}\right),\left(A_{2}^{+} \cap\{3\}\right) \cup\left(A_{3}^{+} \backslash\{3\}\right)\right) \\
& =\mu((\{1\} \cap\{3\}) \cup(\varnothing \backslash\{3\}),(\varnothing \cap\{3\}) \cup(\{1\} \backslash\{3\}),(\{3\} \cap\{3\}) \cup(\{2\} \backslash\{3\}),(\{2\} \cap \\
& \{3\}) \cup(\varnothing \backslash\{3\}))=\mu(\varnothing,\{1\},\{2,3\}, \varnothing) \\
& \mu_{\mathcal{A}}(\varnothing)=\mu\left(\left(A_{2}^{-} \cap \varnothing\right) \cup\left(A_{3}^{-} \backslash \emptyset\right),\left(A_{1}^{-} \cap \emptyset\right) \cup\left(A_{2}^{-} \backslash \emptyset\right),\left(A_{1}^{+} \cap \varnothing\right) \cup\left(A_{2}^{+} \backslash \emptyset\right),\left(A_{2}^{+} \cap \varnothing\right) \cup\left(A_{3}^{+} \backslash \emptyset\right)\right) \\
& =\mu((\{1\} \cap \varnothing) \cup(\varnothing \backslash \emptyset),(\varnothing \cap \varnothing) \cup(\{1\} \backslash \emptyset),(\{3\} \cap \emptyset) \cup(\{2\} \backslash \emptyset),(\{2\} \cap \varnothing) \cup(\varnothing \backslash \emptyset)) \\
& =\mu(\varnothing,\{1\},\{2\}, \varnothing)
\end{aligned}
$$

\section{Appendix C. Derivation of Eq. (11)}

$$
\begin{aligned}
(1)-(2) & =u_{1}^{2}\left[\mu\left(1 \cup C_{1}(1)\right)-\mu\left(C_{1}(1)\right)\right]-u_{1}^{1}\left[\mu\left(1 \cup C_{1}(2)\right)-\mu\left(C_{1}(2)\right)\right] \\
& +u_{2}^{j}\left[\mu\left(2 \cup C_{2}(1)\right)-\mu\left(C_{2}(1)\right)-\mu\left(2 \cup C_{2}(2)\right)+\mu\left(C_{2}(2)\right)\right],
\end{aligned}
$$

where $C_{i}(j)$ denotes the set of criteria whose values are greater than or equal to the value of criterion $i$ in alternative $j$.

- $C_{2}(1)=C_{2}(2)$

This situation occurs when one of the following two rankings is valid:

$$
\begin{array}{ll}
- & u_{2}^{j}>u_{1}^{2}>u_{1}^{1} \\
- & u_{1}^{2}>u_{1}^{1}>u_{2}^{j}
\end{array}
$$

Then, $C_{1}(1)=C_{1}(2)$. Moreover, the third term in Eq. (C.1) vanishes. Therefore,

$$
(1)-(2)=\left(u_{1}^{2}-u_{1}^{1}\right)\left[\mu\left(1 \cup C_{1}(1)\right)-\mu\left(C_{1}(1)\right)\right]
$$

In Eq. (C.2), $C_{1}(1)=\{2\}$ if $u_{2}^{j}>u_{1}^{2}>u_{1}^{1}$ and $C_{1}(1)=\emptyset$ if $u_{1}^{2}>u_{1}^{1}>u_{2}^{j}$.

- $C_{2}(1) \neq C_{2}(2)$

This situation occurs when $u_{1}^{2}>u_{2}^{j}>u_{1}^{1}$. Then, $C_{1}(1)=\emptyset, C_{1}(2)=\{2\}, C_{2}(1)=$ $\{1\}, C_{2}(2)=\emptyset$. Therefore,

$$
(1)-(2)=\left(u_{2}^{j}-u_{1}^{1}\right)[\mu(1,2)-\mu(2)]+\left(u_{1}^{2}-u_{2}^{j}\right)[\mu(1)-\mu(\varnothing)]
$$

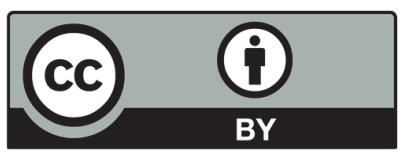

(C) 2020 by the authors; licensee Growing Science, Canada. This is an open access article distributed under the terms and conditions of the Creative Commons Attribution (CC-BY) license (http://creativecommons.org/licenses/by/4.0/). 